\title{
Kernos
}

Revue internationale et pluridisciplinaire de religion grecque antique

$27 \mid 2014$

Varia

\section{Rituels, transmission et savoirs partagés à Éphèse}

Des associations dionysiaques aux communautés chrétiennes

\section{Anne-Françoise Jaccottet et Francesco Massa}

\section{(2) OpenEdition}

Édition électronique

URL : http://journals.openedition.org/kernos/2284

DOI : $10.4000 /$ kernos.2284

ISSN : 2034-7871

\section{Éditeur}

Centre international d'étude de la religion grecque antique

\section{Édition imprimée}

Date de publication : 1 novembre 2014

Pagination : 285-318

ISBN : 978-2-87562-055-2

ISSN : 0776-3824

\section{Référence électronique}

Anne-Françoise Jaccottet et Francesco Massa, «Rituels, transmission et savoirs partagés à Éphèse », Kernos [En ligne], 27 | 2014, mis en ligne le 01 octobre 2016, consulté le 30 avril 2019. URL : http:// journals.openedition.org/kernos/2284 ; DOI : 10.4000/kernos.2284

Ce document a été généré automatiquement le 30 avril 2019.

Kernos 


\title{
Rituels, transmission et savoirs partagés à Éphèse
}

Des associations dionysiaques aux communautés chrétiennes*

\author{
Anne-Françoise Jaccottet et Francesco Massa
}

1 Plusieurs études récentes se sont penchées sur l'évolution ou la diffusion des pratiques cultuelles des Grecs et des Romains, entre tradition et innovation, entre norme et originalité, entre valeurs largement reconnues et particularités locales ${ }^{1}$. S'il est aujourd'hui admis que les rituels ne sont pas immuables, mais bien en constante adaptation, en dialogue et en interaction avec les différentes composantes de la société, reste à poursuivre la mise en lumière - de cas en cas, comme de façon plus générale - du pourquoi et du comment de ces changements, de leurs fondements complexes et de leur moteur au croisement de dynamiques sociales, politiques ou culturelles. S'interroger plus précisément sur la transmission de pratiques ou de termes rituels peut dès lors représenter un biais par lequel reprendre concrètement et ponctuellement ce questionnement fondamental. Cette démarche ne saurait s'envisager autrement qu'en partant d'un cas particulier, d'un dossier ou ensemble de dossiers circonscrits, pour envisager dans un second temps un élargissement spéculatif de la question à des processus plus globaux. C'est le parti pris par cette étude dont Éphèse sera le point focal. C'est en effet autour de la capitale de la province d'Asie que nous pourrons articuler trois dossiers distincts mais qui se répondent et se révèlent complémentaires dans la perspective d'une analyse plus généralisée de la question des savoirs partagés en matière de référence rituelle.

2 Il s'agira dans un premier temps d'étudier le cas des associations dionysiaques, dont Éphèse nous fournit un corpus intéressant. Largement diffusées dans l'espace et le temps, ces associations se caractérisent d'un bout à l'autre de l'Antiquité par l'indépendance dont chacune fait preuve, tant dans sa forme que dans son profil cultuel. Ce trait fondamental se manifeste notamment dans la disparité des fonctions rituelles attestées dans les documents épigraphiques. Chaque association est un cas unique, une création sur mesure, offrant bien souvent des fonctions qui ne se retrouvent dans aucune autre association bachique et proposant toujours une combinaison originale - exclusive - de 
fonctions rituelles ${ }^{2}$. Ces particularités fondamentales soulèvent directement la question des référents à partir desquels se constitue le profil rituel de tel ou tel groupement cultuel : sur quelles bases sont choisies ou créées les fonctions liées au déroulement du culte, sur quelles traditions religieuses ou littéraires, sur quels emprunts, sur quel substrat régional sont construits les rituels associatifs ? Étudier dans cette perspective le cas particulier des associations dionysiaques dans la seule ville d'Éphèse ne saurait épuiser le sujet loin s'en faut. Mais le dossier éphésien permettra de faire entrer en résonance un examen de la dynamique interne à la cité en matière de références religieuses et une approche des échanges que l'on peut mettre en lumière sur le plan régional. Il s'agira en outre de déterminer si ces échanges sont de nature avant tout cultuelle ou culturelle. En effet, la diffusion littéraire et iconographique de la figure de Dionysos et de son univers imaginaire ou rituel ainsi que le rôle de koinè que joue la référence dionysiaque dans les mondes grec et romain sont à même d'alimenter un savoir très général et partagé dans tout l'espace méditerranéen, sans forcément de lien direct avec une pratique rituelle quelconque; le cultuel se colore ainsi potentiellement de culturel, selon nos catégories modernes de pensée.

3 C'est dans cette optique, que nous convoquerons, dans un second temps, le témoignage d'Ignace d'Antioche, un des premiers auteurs chrétiens dont nous ayons conservé les écrits, et dont la Lettre aux Éphésiens se révèle riche en échos de traditions cultuelles ou associatives. Cette écriture en résonance avec des réalités rituelles non chrétiennes ouvre notamment de nouvelles perspectives dans l'analyse de la circulation d'un vocabulaire et de concepts traditionnels : savoir rituel véritablement partagé ou koinè culturelle utilisée métaphoriquement pour les besoins d'une communication efficace sur la base de référents que tout un chacun connait ? Les allusions à des évidences rituelles effectives et bien connues d'un Ignace comme des Éphésiens à qui il adresse sa lettre sont-elles à comprendre également comme un moyen de pallier l'absence d'un vocabulaire de référence chrétien au tout début du $\mathrm{II}^{\mathrm{e}}$ siècle de notre ère?

4 La reprise, dans un troisième volet, d'une inscription mettant en scène le hiérophante public d'Éphèse et révélant son rôle primordial dans la transmission des spécificités rituelles auprès des magistrats qui se succèdent dans les sacrifices officiels ouvrira le débat sur les acteurs et les modalités de transmission d'un certain savoir pratique en matière rituelle.

5 C'est sur la base de la mise en parallèle des trois dossiers examinés que pourront être tentées, en guise de conclusion, des hypothèses sur les moteurs des échanges, transmissions ou innovations rituels, permettant une réflexion sur ce que recouvre in fine la notion de savoir partagé en matière cultuelle.

\section{Les associations dionysiaques éphésiennes}

6 Les associations dionysiaques d'Éphèse, qui nous sont perceptibles par le biais de onze inscriptions ou fragments d'inscriptions, offrent un terrain d'analyse fécond à deux points de vue pour évaluer les solutions pratiques et les outils référentiels convoqués en matière de tradition ou transmission cultuelles. La dimension associative premièrement induit une part de création dans l'instauration et la gestion d'un culte qui tient lieu de ciment à ces microcosmes sociétaux. La référence dionysiaque ensuite permet de questionner les relations entre référents culturels et proprement cultuels, par l'ancrage de la figure de Dionysos non seulement dans la sphère rituelle mais bien plus largement 
dans les récits, dans les images et tout ce qui nourrit cette koinè dionysiaque que partagent Grecs et Romains.

7 Afin de replacer l'analyse dans le contexte conceptuel antique, il n'est pas inutile de commencer par interroger l'imaginaire de l'installation d'un nouveau culte. C'est en effet la nouveauté qui requiert le plus directement un discours sur la coutume, sa transmission et la légitimité du rite instauré, questions particulièrement de mise dans le cadre des associations dionysiaques. Un document bien connu de Magnésie du Méandre nous permet de percevoir ce discours à propos de l'instauration du culte dionysiaque dans la cité.

C'est par une inscription du II $^{\mathrm{e}}$ siècle de notre ère qui retranscrit un oracle pythique censé avoir été rendu à époque hellénistique à propos de l'instauration du culte de Dionysos dans la cité que nous pouvons entrer dans la dialectique de la nouveauté rituelle et ses présupposés en matière de transmission de rites légitimes et bien fondés ${ }^{3}$. Suite à un

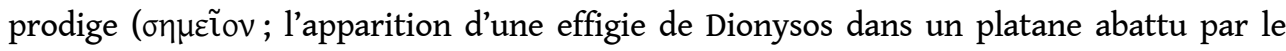
vent), l'oracle de Delphes est consulté par une délégation de Magnètes, dont l'inscription précise le nom par souci d'accréditer la démarche officielle, réelle ou présentée comme telle ${ }^{4}$. La réponse du dieu donne les raisons de l'apparition tout comme les moyens d'agir pour répondre à ce signe pour le bien de la communauté (1. 19-30).

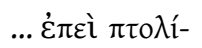

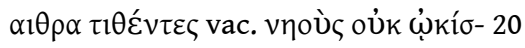

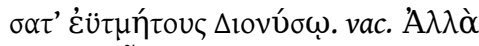

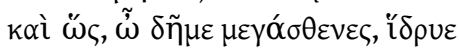

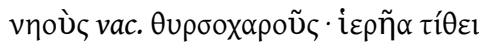

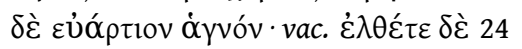

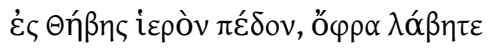

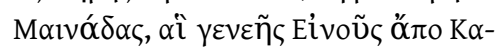

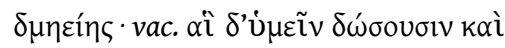

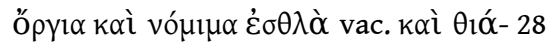

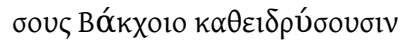

ह่v ö $\sigma \tau \varepsilon$.

[...] C'est que, lorsque vous avez bâti votre ville, vous avez négligé d'édifier des temples en belle place à Dionysos, - alors voici, peuple magnanime, - élève des temples qui prennent plaisir aux thyrses, mets ici un digne et saint prêtre, - et puis allez en la terre sacrée de Thèbes, où vous prendrez des Ménades de la race d'Inô la Cadméenne. - Elles vous transmettront les orgia et les bonnes traditions et fonderont des thiases de Bakkhos dans la ville [...].

9 Le signe que représente l'apparition de l'effigie de Dionysos dans le platane renvoie à un manque cultuel et la solution passe bel et bien par l'instauration d'un culte que les «fondateurs » avaient négligé au moment d'installer la ville. Mais sur quelles bases et selon quelles modalités instaurer ce culte nouveau? Selon le modèle théorique, ou mythe étiologique livré par l'inscription, il ne suffit pas de construire un temple et d'y installer officiellement un prêtre pour qu'un culte nouveau puisse véritablement fonctionner. Il faut une autorité reconnue qui transmette les bons rites, ceux dont l'origine garantira la légitimité et l'efficience religieuse. L'oracle envoie les Magnètes à la source même du dionysisme, à Thèbes, pour trouver les trois femmes ${ }^{5}$ aptes à transmettre les ópyla - à prendre probablement ici dans leur sens matériel ${ }^{6}$-, ainsi que les « bonnes traditions » vó $\iota \mu \alpha \dot{\varepsilon} \sigma \theta \lambda \alpha$-, et qui fonderont encore chacune un thiase.

Si l'on analyse les données de l'inscription dans l'optique de la transmission nécessaire à une fondation légitime et fonctionnelle, on constate que ce que les Magnètes, et l'oracle 
qui les oriente, attendent des trois Ménades thébaines touche trois domaines distincts et complémentaires - de compétence :

- la transmission d'objets (őpyı), durables ou périssables, équivalant au «nécessaire du bon prêtre ou de la bonne prêtresse ", soit ce qui, matériellement, va rendre les rites possibles et efficaces ;

- la transmission des traditions ( $($ ó $\mu \mu \alpha)$, des règles, qui régissent l'usage des lieux et des choses: la bonne périodicité, les bonnes formules, le savoir-faire, qui assurent le bon déroulement des cérémonies et en donnent peut-être aussi le fondement, le récit

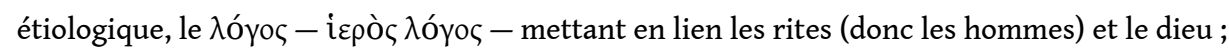

- la transmission d'un système d'organisation communautaire, par la fondation de thiases, d'organisations rituelles relevant d'une compétence à la fois religieuse et institutionnelle.

11 La légitimité de cette triple compétence attendue des Ménades est cautionnée par leur origine, elle-même cautionnée par l'oracle: Thèbes, que l'oracle qualifie même de «terre sainte (į́pòv $\pi \varepsilon ́ \delta o v)^{7}$ ». Thèbes, berceau de Dionysos et de son culte; mais plus précisément encore, des femmes « de la race d'Inô la Cadméenne ", des Thébaines qui, par leur sang, par leur lignée, sont considérées comme héritières des bonnes traditions. Une hérédité biologique pour un héritage rituel et religieux.

Que cet oracle ait été effectivement rendu par Delphes ou non ${ }^{8}$, il nous dévoile l'imaginaire lié à la fondation d'un culte et les référents idéaux recherchés pour légitimer les pratiques rituelles nouvelles au sein d'une cité. L'analyse de l'inscription de Magnésie fait apparaître les traces d'une mémoire littéraire qui agit dans la construction de l'oracle pythique en faisant des trois femmes thébaines, filles de Cadmos, les gardiennes des « bonnes traditions » dionysiaques ${ }^{9}$; mémoire littéraire, ou peut-être, en amont, mémoire d'un imaginaire fort, dont les textes sont des relais autant que des moteurs, tête émergée, à nos yeux, d'un iceberg de tradition. Dès l'époque classique, les récits liés à Dionysos avaient contribué à construire et à diffuser l'image de Thèbes comme lieu fondateur de son culte en Grèce : assurément, les Bacchantes d'Euripide qui mettaient en scène l'arrivée du dieu à Thèbes et l'initiation forcée des femmes de la cité, embrigadées en trois thiases, ont eu un rôle important en fonction de leur large diffusion comme texte scolaire et comme pièce prisée du répertoire ${ }^{10}$. On aurait tort pour autant de voir dans cette tragédie le seul référent littéraire, le seul jalon de cette tradition discursive ; la littérature à sujet dionysiaque était très riche et d'autres textes, rédigés dans d'autres lieux et d'autres temps, pouvaient entrer dans l'imaginaire des associations dionysiaques. Il suffit de penser, à titre d'exemple, à l'Idylle XXVI de Théocrite, qui insiste dès les deux premiers vers sur le nombre « trois» :

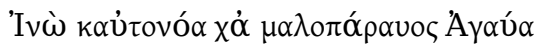

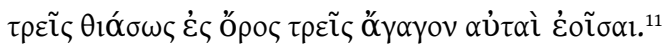

Inô, Autonoé et Agavé aux joues candides

trois thiases conduisirent vers les monts et elles-mêmes étaient trois.

13 Trois thiases conduits «vers les monts » ( $\dot{\varepsilon} \zeta$ ópoৎ) par les trois sœurs, filles de Cadmos, qui préparent des offrandes et les déposent sur les autels, « comme l'enseignait Dionysos

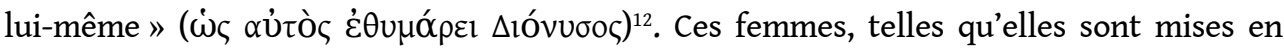
scène, connaissent la volonté du dieu, la respectent et la transposent dans le déroulement $\mathrm{du}$ rituel ${ }^{13}$. Elles sont ainsi présentées comme les dépositaires de la tradition originelle qu'elles détiennent en ligne directe du dieu lui-même, leur parent. Elles sont donc le prototype de l'agent parfait de transmission légitime du rituel dionysiaque. En installant à Magnésie, pour les besoins de la fondation du culte de Dionysos, trois «Ménades 
thébaines de la race d'Inô la Cadméenne ", les Magnètes, en suivant l'oracle delphique, s'assurent ainsi un lien rituel on ne peut plus légitime, du moins en référence à l'imaginaire généralement admis, à un consensus culturel tablant sur une koinè dionysiaque.

Le contenu de l'inscription de Magnésie n'est vraisemblablement qu'une fiction étiologique de la fondation du culte dionysiaque dans la cité ${ }^{14}$. Une telle mise en scène a cela de précieux qu'elle trahit la façon de concevoir in abstracto la transmission parfaite d'un ensemble de compétences, garanties par leur origine, nécessaires à la fondation d'un culte. On a là, en somme, la théorie, le cas idéal du transfert d'un 'savoir religieux' complet en toute légitimité, une sorte de 'kit de savoir-faire dionysiaque'. Qu'en est-il alors dans la réalité ? À quelle "source » les associations dionysiaques, confrontées à ce même problème pratique de transmission de rites et de «bonnes traditions", vont-elles puiser pour forger leurs rites et leur vocabulaire cultuel?

Étant donné les spécificités des documents qui portent témoignage des associations, c'est par l'examen des titres de fonctions rituelles des divers groupements dionysiaques que l'on peut espérer approcher les mécanismes de référence à l'œuvre dans l'institution des cultes associatifs ${ }^{15}$. Une liste des titulatures et fonctions, rencontrées dans les associations dionysiaques éphésiennes, permettra de dresser un premier tableau de la situation. Elles sont répertoriées ci-dessous selon un ordre chronologique et sous une forme "décontextualisée », au nominatif, et nominalisées ${ }^{16}$, afin de faciliter une perception globale de la question.

IEph 33298 (JACCOTTET no 135) Trajan (de Thyaira)

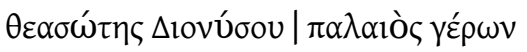

IEph 275 (JACсотTET $n^{0} 136$ ) 119-129 de notre ère

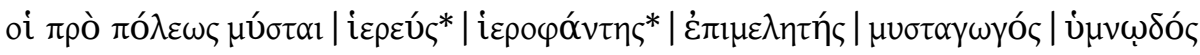

IEph 1601-1603 + 1268 (JACCOTTET $\left.n^{\circ} 137-139\right)$ Hadrien

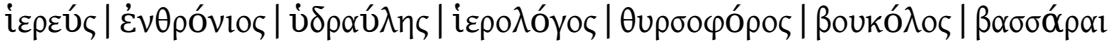

IEph 1595 (JACCOTTET no 140) Marc-Aurèle-Commode

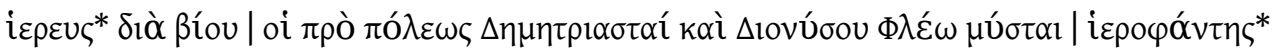

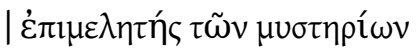

IEph 1211 (JACCOTTET $n^{\circ}$ 141) date incertaine

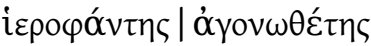

IEph 293 (JACCOTTET $\mathbf{n}^{\circ}$ 142) Commode

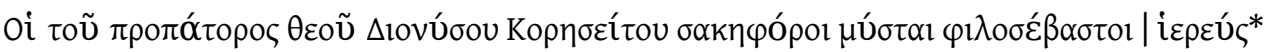

IEph 1267 (JACсотTET $n^{\circ}$ 134) fin du II ${ }^{e}$ siècle de notre ère

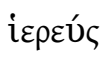

Ce premier parcours des inscriptions dionysiaques éphésiennes surprend peut-être en nous faisant constater que les références explicites au monde dionysiaque sont rares dans les fonctions rituelles éphésiennes; ce phénomène se remarque d'ailleurs de façon générale dans l'ensemble des associations dionysiaques, toutes régions et époques confondues. La tradition clairement exprimée dans l'instauration du culte dionysiaque à Magnésie ne transparaît ainsi que fort peu dans la réalité des fonctions associatives

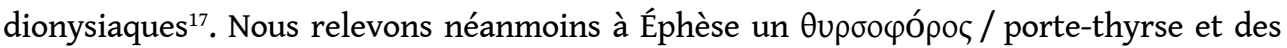


$\beta \alpha \sigma \sigma \alpha \alpha_{\rho} \alpha_{1} /$ bacchantes thraces à la peau de renard. Ces deux titulatures à connotation bachique transparente proviennent d'un groupement dionysiaque d'époque hadrienne, connu par plusieurs inscriptions fragmentaires trouvées dans le théatre, qui donnent la liste des participants aux sacrifices rassemblant plusieurs fois par an cette communauté1 ${ }^{18}$. Les mêmes fonctions reviennent donc régulièrement, endossées parfois par des personnes différentes. Cette constatation permet de voir, dans les fonctions que nous pouvons relever, des cadres fixes en relation avec le rituel et non des titres attribués ad personam pour honorer tel ou tel individu de marque ${ }^{19}$.

Ces deux fonctions renvoient à une tradition bachique très largement partagée et répandue; inutile d'insister sur le thyrse qui s'impose de façon flagrante tant dans les récits dionysiaques que dans les images et qui est une composante centrale de

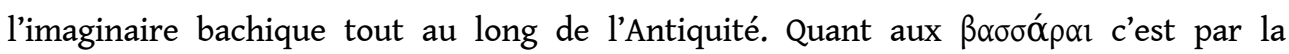
tradition littéraire qu'elles nous sont connues. Nous savons, par exemple, qu'Eschyle avait écrit une tragédie portant ce titre et relatant vraisemblablement les mésaventures d'Orphée mis en pièce par ces bacchantes thraces ${ }^{20}$; en outre, au $\mathrm{I}^{\mathrm{er}}$ ou au $\mathrm{II}^{\mathrm{e}}$ siècle de notre ère, Dionysios, poète dont nous ne connaissons presque rien, fut l'auteur d'une œuvre appelée Bassarika ${ }^{21}$.

Dans ce contexte il ne peut être que frappant de constater que le titre de thyrsophore, tout évident qu'il soit dans une logique dionysiaque, n'apparaît dans aucune autre association dionysiaque connue à ce jour. En revanche, une autre inscription éphésienne ${ }^{22}$ mentionne la dédicace de thyrses par un hiérophante; à nouveau, il s'agit de la seule dédicace de thyrses connue. Quant aux $\beta \alpha \sigma \sigma \alpha ́ \alpha \alpha 1$, on en trouve la trace indirecte à

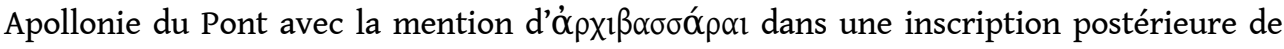
plus d'un demi-siècle ${ }^{23}$, et dans le cosiddetto Thiase de Torre Nova (ou du Metropolitan Museum) qui compte deux $\alpha$ $\rho x ı ß \sigma \sigma \sigma \alpha ́ \rho o l$, fort bien placés hiérarchiquement au sein des 26 fonctions que révèle cette association, postérieure elle aussi, au témoignage éphésien ${ }^{24}$. La rareté des références à l'univers dionysiaque communément diffusé et connu de tout un chacun est ainsi une donnée manifeste du monde associatif dionysiaque, et Éphèse, tout en entrant dans cette dynamique, se révèle peut-être un peu plus perméable à la tradition « imaginaire».

Devrait-on citer, parmi les fonctions qui portent en elles une référence dionysiaque, les ßoukóגol, ou bouviers, qu'atteste ce même groupement qui se rassemblait périodiquement dans le théâtre ou à proximité immédiate ? Nous sommes là face à un cas particulier, face au développement d'une nouvelle fonction dionysiaque sur la base d'un substrat indépendant au départ : les ßoukódor, liés de façon lâche et sporadique au monde dionysiaque durant plusieurs siècles, apparaissent comme une véritable fonction rituelle bachique à la fin $d u ~^{\text {er }}$ siècle avant notre ère. Il n'est pas nécessaire d'expliciter ici l'imbroglio religieux, littéraire et imaginaire qui, d'un Dionysos taureau en passant par la marginalité des zones fréquentées tant par les bouviers que par les thiases bachiques et en prenant au passage toute la dimension idyllique du monde agro-pastoral a abouti à l'adéquation rituelle d'une fonction de bouviers avec le culte dionysiaque ${ }^{25}$. Il suffira de constater que de nombreux indices laissent penser que Pergame a été le lieu probable de l'invention de cette nouvelle fonction rituelle. C'est là que les bouviers sont devenus véritablement dionysiaques, qu'ils sont devenus une fonction rituelle nouvelle appelée à connaître un essor important dans le cadre associatif bachique. Si les bouviers dionysiaques apparaissent de façon certaine, quoiqu'indirecte par l'entremise du titre d' archiboukolos, à Pergame dès 27 avant notre ère avec deux autels parallèles dédiés par un 


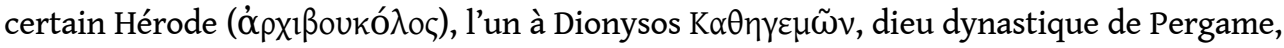
l'autre à Auguste ${ }^{26}$, il est intéressant de suivre la diffusion de cette fonction dans le temps et l'espace ${ }^{27}$. Prenons tout d'abord l'ensemble des témoignages de cette fonction liturgique (Fig. 1, légende donnée en note) ${ }^{28}$.

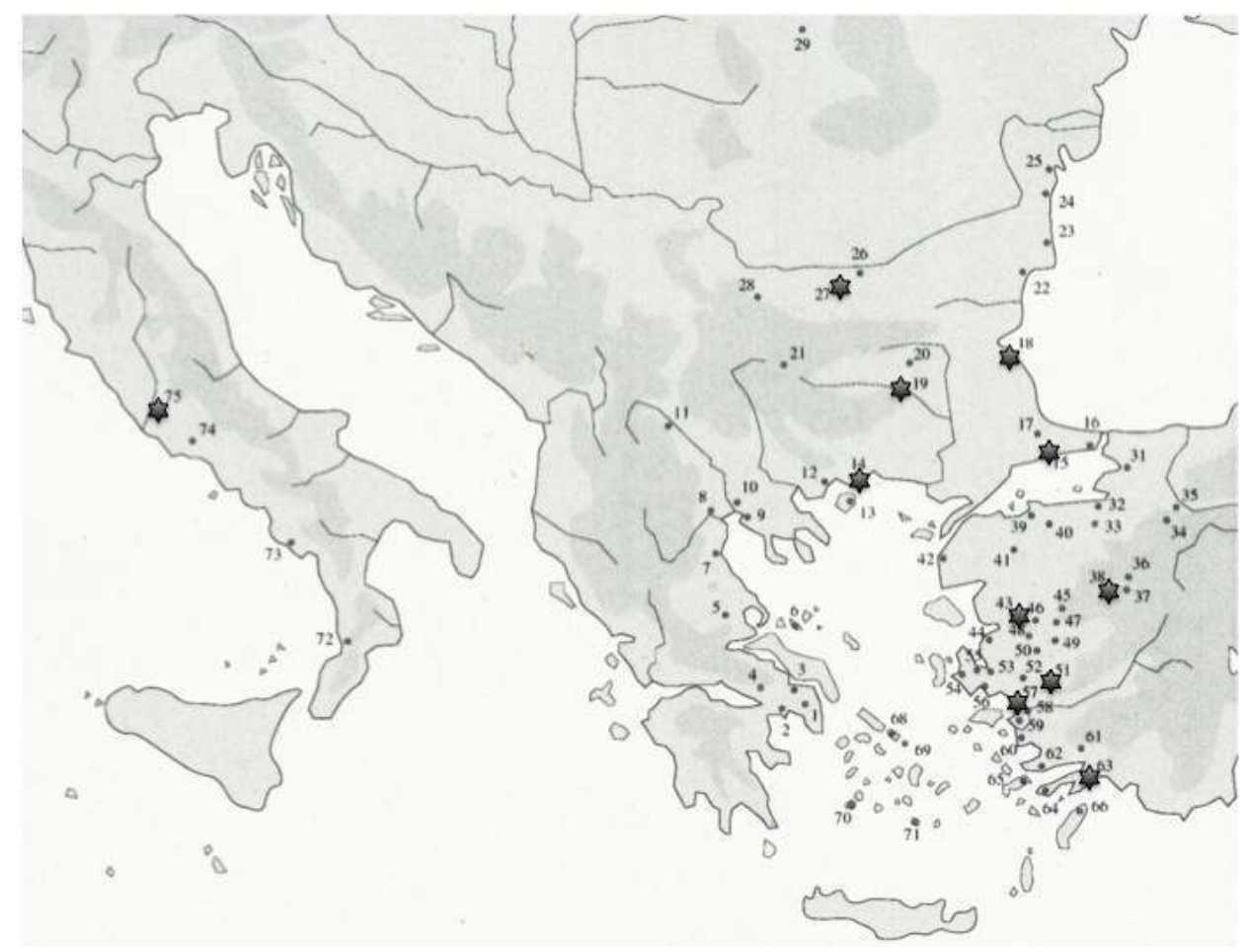

Figure 1

On perçoit aisément l'ancrage micrasiatique du titre, avec un développement sur la rive gauche du Pont-Euxin débordant légèrement en Thrace et Mésie. Quelques occurrences sortent de ce cadre, comme par exemple la fameuse association dite de Torre Nova, aux portes de Rome, vers 160 de notre ère, dont les racines mytiléniennes sont bien connues ${ }^{29}$ ; la reprise par l'aristocratie sénatoriale de Rome entre 376 et 385 de notre ère, du terme d'archibucolus, dans des inscriptions tauroboliques et à côté d'autres emprunts à des cultes divers, montre quant à elle que le titre avait acquis une renommée rituelle suffisante pour entrer dans ces constructions philosophico-piétistes mêlant les fonctions issues de divers cultes $^{30}$. Une analyse chronologique différenciée des différents témoignages apporte une dimension supplémentaire. Une mise en scène visuelle sera plus parlante que tout commentaire (Fig. 2). 


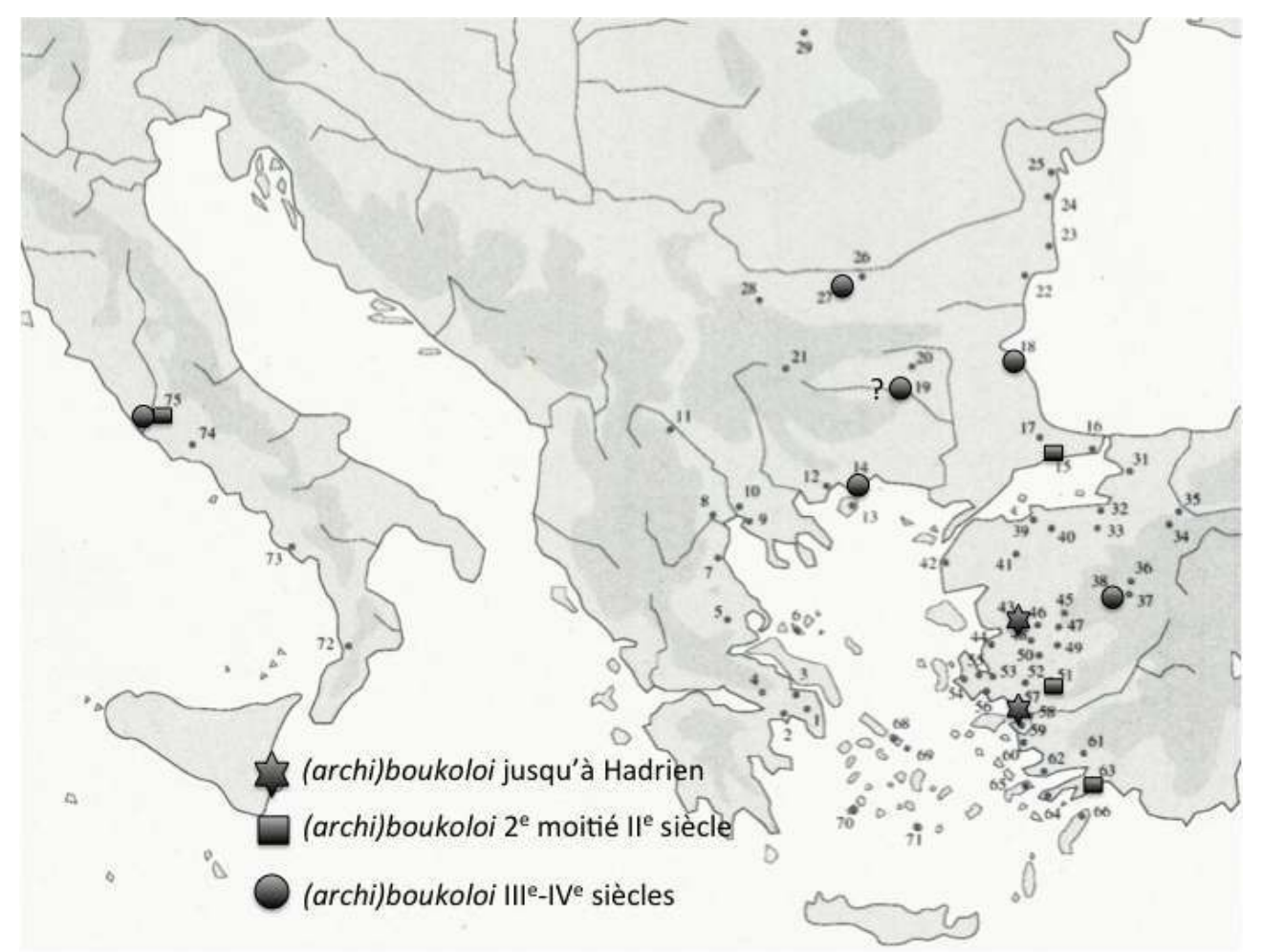

Figure 2

Avec l'attestation à Éphèse de bouviers dans ce groupement cultuel d'époque hadrienne, la ville d'Artémis se présente comme la seconde, après Pergame, à adopter cette nouveauté rituelle, avant sa large diffusion à partir de la seconde moitié du II siècle. Hasard des découvertes ou rapprochement pertinent? Nous pencherons pour la seconde hypothèse. Le lien entre les bouviers pergaméniens et ce groupement éphésien est d'autant plus manifeste que les deux entités semblent entretenir des liens très serrés avec le théâtre, trait qui ne s'impose absolument pas dans les autres attestations postérieures, autant que notre documentation nous permette d'en juger. À Pergame, au travers des huit inscriptions, qui s'étendent sur quelque 140 ans, entre 27 avant notre ère et l'époque d'Hadrien, nous voyons apparaître plusieurs fonctions liées à des exécutions scéniques. Dès la première moitié $\mathrm{du} \mathrm{I}^{\mathrm{er}}$ siècle de notre ère, les bouviers, terme qui donne son titre à l'association, comptent en leurs rangs un $\chi 0 \rho \eta \gamma o ́ \varsigma^{31}$; puis, apparaît vers la fin du siècle un

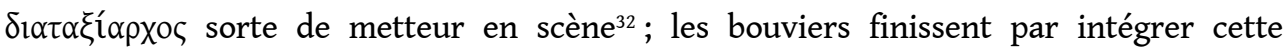
composante orchestique à leur titulature même, en devenant dès le début du $\mathrm{II}^{\mathrm{e}}$ siècle, les

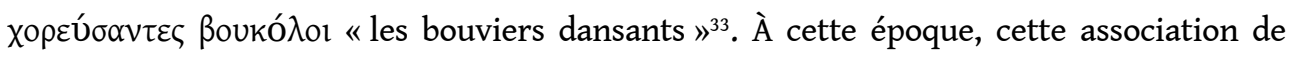
bouviers est placée sous l'égide directe du prêtre officiel de Dionysos à Pergame : intégrés aux célébrations triétériques de la cité pour Dionysos, ils célèbrent, par leur danses et autres manifestations "théâtrales" les "divins mystères " pergaméniens. Ces bouviers pergaméniens étaient ainsi une composante bien visible, en grande partie publique, de la vie cultuelle dionysiaque de la ville. C'est vraisemblablement cette visibilité, mise en scène dans le théâtre, qui a inspiré le groupement éphésien, dont les réunions se tenaient également dans le théâtre ou à proximité immédiate.

Au travers de l'adoption par un groupe éphésien de la fonction de bouviers, on observe donc la diffusion directe, croyons-nous, d'une nouveauté rituelle d'une association à une 
autre, d'une ville à une autre. Ce transfert ne se fait pas selon un axe vertical de transmission de savoirs par la tradition, mais selon un axe horizontal, par émulation, émanant directement d'un partage d'expérience, dans une parfaite contemporanéité. On ne peut éviter de placer cette « contagion » dans le cadre plus large des rivalités qui ont animé dès le $\mathrm{I}^{\mathrm{er}}$ siècle de notre ère les deux cités, placées en position de concurrence directe et constante pour l'obtention du statut très envié de ville néocore ${ }^{34}$. On remarquera, dans ce contexte, que les bouviers pergaméniens - les premiers bouviers dionysiaques - apparaissent dès Auguste dans une position très manifeste d'allégeance au pouvoir romain, avec la dédicace de l'autel à Auguste par l'ópxıßoukó入oc. Et les associations éphésiennes, avec ou sans bouviers, ne sont pas en reste en ce qui concerne les honneurs rendus aux empereurs, comme en témoignent trois inscriptions dionysiaques de la ville : la première honore Trajan en lui accordant le titre de thiasote de

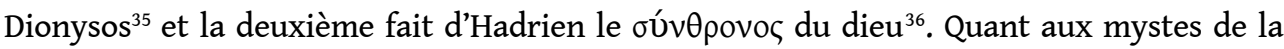

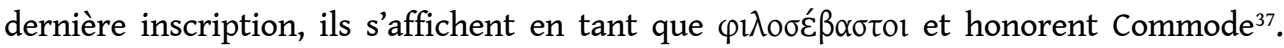
Dans ce contexte d'émulation concurrentielle, ce n'est donc pas un hasard si c'est à Éphèse qu'apparaît le premier surgeon des bouviers pergaméniens.

S'il y a manifestement adoption d'une nouveauté rituelle dionysiaque créée ou développée à Pergame, on aimerait pouvoir préciser la nature et les contours de cette transmission, par émulation. Derrière le titre, quel contenu rituel est-il effectivement transmis ? A-t-on affaire à une imitation de façade ou à une adoption d'un concept rituel complet? La nature des documents disponibles ne nous permet malheureusement pas d'aller plus loin que la constatation d'un même lien au théâtre. On relèvera néanmoins une différence notable: si les bouviers pergaméniens sont une association en tant que telle, le titre de bouvier n'apparaît qu'au singulier dans le groupement d'Éphèse, endossé à chaque réunion par un, éventuellement deux participants aux sacrifices. Ce passage, de Pergame à Éphèse, d'une dénomination collective qui donne son nom à l'association, à une fonction isolée au sein d'un groupe qui connaît d'autres fonctions sans que celles-ci ne lui soient subordonnées ne saurait pour autant croyons-nous représenter un argument pour réfuter la «filiation». Cela dénote bien plus la souplesse de mise dans l'adoption, même directe, d'une titulature et de la fonction qui lui est liée.

31 Si l'on reprend la liste des fonctions révélées par les différents fragments du théâtre qui mettent en scène ces boukoloi ${ }^{38}$, on relève, à côté du thyrsophore et des $\beta \alpha \sigma \sigma \alpha ́ \alpha \alpha 1$ déjà évoqués, la présence d'un prêtre (iॄp£úc), fonction dont la banalité ne nous dira rien des orientations cultuelles du groupe, mais aussi celle d'un joueur d'orgue hydraulique

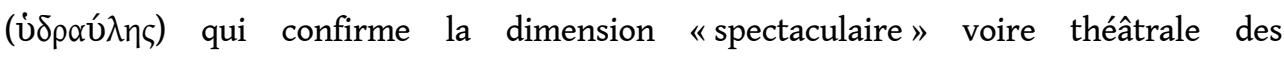
manifestations cultuelles de ce groupe. Restent encore deux autres fonctions énigmatiques, sans parallèle ni dans les associations dionysiaques ni dans aucune

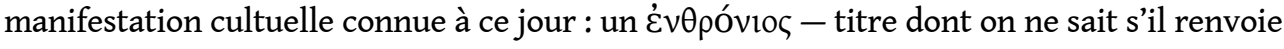
à un rite d'intronisation rituelle ou à une simple marque honorifique - et, apparaissant

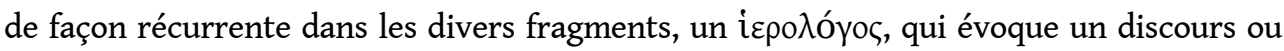
un récit sacré dont on aimerait pouvoir préciser la teneur et la fonction. On connaît des theologoi, mais le hierologos est un hapax dans la sphère cultuelle "païenne $"^{39}$. Les textes, en revanche, témoignent de l'existence de hieroi logoi liés aux pratiques rituelles des cultes à mystères ${ }^{40}$. Hérodote emploie l'expression à plusieurs reprises dans son deuxième livre consacré à l'Égypte, autant pour définir des logoi égyptiens que des logoi dévoilés pendant des cérémonies du monde grec $^{41}$. Pausanias lui-même utilise l'expression au singulier pour indiquer un récit sacré concernant l'interdiction de se nourrir de fèves, 
pratique répandue chez les Phénéates dans l'initiation ( $\tau \varepsilon \lambda \varepsilon \tau \eta ́)$ de Déméter ${ }^{42}$. Hérodote comme Pausanias se réfèrent vraisemblablement à des discours oraux, des récits « sacrés » dont il faut garder le secret en raison de leur contenu lié aux pratiques rituelles initiatiques $^{43}$.

Une lecture maximaliste de cette fonction, à la fois comme signe de l'élaboration d'un hieros logos et de sa récitation rituelle dans une acception performative, pourrait se situer au point de convergence entre un savoir de type théorique et un savoir-faire de type rituel ; mais cela reste une hypothèse et une piste de réflexion.

Le cas de ce groupe sacrificiel éphésien est particulièrement intéressant pour l'étude de la constitution d'une entité rituelle et des présupposés qui en forment la base. On peut en effet décomposer les différentes fonctions constitutives du profil rituel de ce groupe en plusieurs types d'influence et de référents. Le thyrsophore et les $\beta \alpha \sigma \sigma \alpha ́ \alpha \alpha$ nous entraînent du côté d'une influence littéraire ou, plus généralement, de l'adéquation à un modèle globalisé d'imaginaire dionysiaque. Le ßoukó $\lambda$ os trahit l'assimilation en ligne directe d'un modèle nouveau, issu de la ville, proche et rivale, de Pergame et qui doit son adoption peut-être autant à des questions de prestige, voire de politique, qu'à un fondement strictement religieux. Le prêtre s'inscrit dans le modèle le plus général de la ritualité

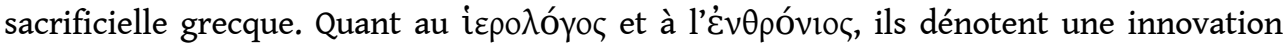
originale, unique, à la fois indispensable et constitutive de l'identité rituelle propre à ce groupement si l'on en croit la permanence de ces fonctions au fil des documents.

Nous poursuivrons l'enquête en mettant en lumière les rapports et partages décelables entre les associations dionysiaques éphésiennes et les autres cultes de la ville. Si des interactions sont susceptibles d'être mises en avant entre différents groupes cultuels dédiés à des divinités différentes, il peut s'avérer pertinent de scruter les modalités de partage, d'échange ou au contraire de mise en avant des spécificités individuelles au moment d'une entrée en relation directe. Une inscription permet d'aborder cette question de la façon la plus concrète ${ }^{44}$.

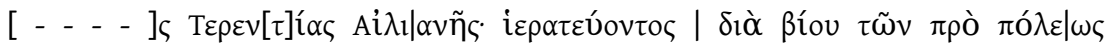

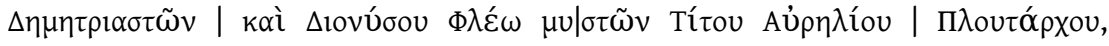

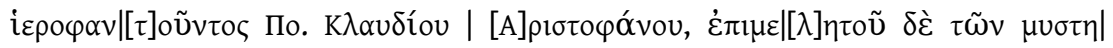

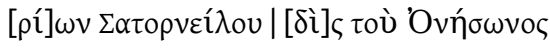

[...] Terentia Aelianè ; Alors qu'était prêtre à vie des Démétriastes pro poleôs et des mystes de Dionysos Phleus T. Aurelius Ploutarkhès, qu'était hiérophante P. Claudius Aristophanès, qu'était épimélète des mystères Satorneilos, fils et petit fils d'Onèson.

Ce document atteste l'existence d'une association qui regroupe sous un même chapeau cultuel, ou du moins sous une même prêtrise, des Démétriastes et des mystes de Dionysos. Telle que l'inscription, malheureusement fragmentaire, la laisse apparaître, l'entité double est en effet placée sous l'autorité commune d'un prêtre, nommé à vie. Faut-il ne voir ici que l'attestation d'une prêtrise attribuée séparément mais conjointement par chacune des deux associations à ce T. Aurelius Ploutarkhès? En d'autres termes, l'association des deux entités ne se fait-elle que par le hasard des circonstances ponctuelles autour et par la figure de ce Ploutarkhès, prêtre à la fois des Démétriastes et des mystes de Dionysos Phleus, par le bais d'un cumul souvent attesté de prêtrises? Cette lectio facilior nous semble peu crédible au vu de la suite de l'inscription: deux autres fonctions sont mentionnées, celle d'hiérophante et celle d'épimélète des mystères, sans qu'il soit spécifié qu'elles ne concernent que l'une ou l'autre des associations et qui, en l'état de l'inscription, semblent se rapporter conjointement aux deux entités. Le caractère 
fragmentaire du document laisse il est vrai planer un dernier doute. Aurions-nous une inscription, honorifique par exemple, proposée soit par les Démétriastes, soit par les mystes de Dionysos Phleus, origine que le début perdu du texte rendrait claire, avec la mention de la double prêtrise de Ploutarkhès, par simple souci de donner à ce personnage tous ses titres? On remarquera que le libellé des deux groupes prend une forme très unitaire ne serait-ce que par l'absence d'un $\tau \tilde{\omega} v$ devant le second groupe ( $\Delta$ ıvv́øov $\Phi \lambda \varepsilon ́ \omega$ $\mu \nu \sigma \tau \tilde{\omega} v)$. Simple oubli du lapicide? Ou formule allégée pour éviter toute redondance ? Si les deux entités étaient clairement distinctes et ne connaissaient de lien que par l'entremise de la double prêtrise de Ploutarkhès, et si donc l'inscription n'émanait que d'une seule de ces deux associations, les deux groupes nominaux auraient probablement été bien circonscrits chacun avec un déterminant marquant la césure et l'identité propre. On a bien plutôt l'impression que les deux groupes sont liés au point peut-être de partager une même qualification de pro poleôs, formule qui pourrait être une «mise en évidence » syntaxique tout comme le déterminant $\tau \tilde{\omega} v$. Nous retiendrons donc la lectio difficilior qui consiste à voir dans cette inscripiton une attestation de la liaison fonctionnelle de deux associations dédiées à des divinités distinctes et liées incontestablement par un prêtre commun.

Se pose alors très directement, dans cette hypothèse de lecture, la question du partage du rituel et des traditions respectives des Démétriastes et des mystes dionysiaques. Doit-on imaginer que les rites mystériques évoqués par l'intermédiaire des fonctions de hiérophante et d'épimélète des mystères sont bel et bien les mêmes, pratiqués en commun par les mystes de Déméter et ceux de Dionysos? Le libellé de l'inscription, qui donne un seul hiérophante et un seul épimélète, tout comme il ne donne qu'un seul prêtre à l'ensemble, pourrait orienter vers cette interprétation ${ }^{45}$. Mais on pourrait également envisager que le hiérophante et l'épimélète interviennent successivement dans des rites mystériques séparés dédiés spécifiquement à Déméter d'une part et de l'autre à Dionysos. Quoi qu'il en soit, dans les deux cas, c'est bien de la mise en commun d'un certain savoir religieux qu'il s'agit. Soit les rites mystériques des Démétriastes et des mystes de Dionysos sont harmonisés pour ne former qu'un seul ensemble rituel cohérent; on aurait alors affaire à un partage des connaissances rituelles qui concerne la communauté associative dans son ensemble. Soit ce partage se situe au niveau des seuls responsables des cérémonies, le hiérophante et l'épimélète, qui eux cumuleraient les savoirs rituels des deux divinités et les proposeraient, de manière distincte et comme 'étanche', aux uns et aux autres. La transmission des savoirs rituels ne serait alors pas élargie à l'ensemble de la communauté, mais restreinte à des spécialistes, capables de décliner les rituels mystériques selon les coutumes des uns comme des autres.

Si l'on ne peut exclure aucune des deux hypothèses, la première, à savoir une mise en commun des rites des deux communautés, nous paraît plus vraisemblable. On constate en effet que l'association des Démétriastes, en tant qu'association indépendante, est active à Éphèse dès la première moitié $d u \mathrm{I}^{\mathrm{er}}$ siècle de notre ère, avec les honneurs rendus aux deux fils de Drusus le jeune ${ }^{46}$. Dès cette époque, ils se présentent comme des Démétriastes $\pi \rho o ̀ ~ \pi o ́ \lambda \varepsilon \omega \varsigma$, dénomination qui reste ancrée dans l'identité du groupe jusqu'à la seconde moitié du $\mathrm{II}^{\mathrm{e}}$ siècle avec le document qui les lie aux mystes de Dionysos. Quant à ces derniers, peut-être en avons-nous une trace, sous forme également indépendante, dans

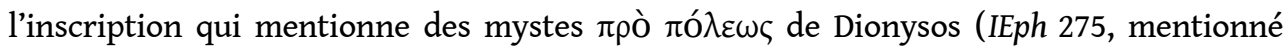
supra dans la liste). Certes le libellé ne précise pas que les rites se font pour honorer un Dionysos $\Phi \lambda \varepsilon \dot{\varepsilon} \zeta$, et l'on connaît d'autre part la diffusion de ce qualificatif exprimant 
davantage la protection apportée par la divinité sur la cité que la localisation géographique du culte ${ }^{47}$. Mais la présence conjointe d'un hiérophante et d'un épimélète rapproche très directement ces mystes dionysiaques de l'association mixte de l'inscription qui nous occupe ici. Nous avons en effet proposé plus haut de considérer le $\pi \rho o ̀ ~ \pi o ́ \lambda \varepsilon \omega \varsigma \zeta$ comme qualifiant autant les Démétriastes que les mystes de Dionysos $\Phi \lambda \varepsilon v ́ \varsigma$, dans une mise en évidence syntaxique. D'autre part, une autre inscription éphésienne du tout début du $\mathrm{I}^{\text {er }}$ siècle de notre ère montre la proximité effective des cultes de Dionysos $\Phi \lambda \varepsilon \dot{\varepsilon} \varsigma$ et des déesses éleusiniennes ${ }^{48}$.

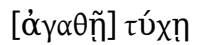

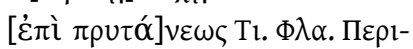

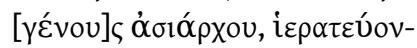

$$
\begin{aligned}
& \text { [ }
\end{aligned}
$$

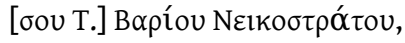

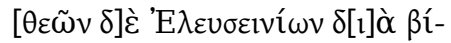

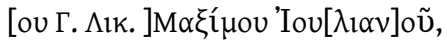

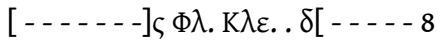

$$
\begin{aligned}
& \text { [-.....-- ].o.[- - - }
\end{aligned}
$$

Chacun des cultes dispose de son propre prêtre et l'on ne saurait encore parler de fusion. Mais ce rapprochement entre le prêtre de Dionysos Phleus et celui des déesses éleusiniennes montre les affinités entre les deux cultes et rend plausible un développement vers une union des deux entités et une mise en commun des rites mystériques, dans un véritable travail sur le savoir rituel partagé.

Quel que soit le modèle de partage qui a prévalu, retenons la possibilité pour deux cultes clairement distincts à l'origine, comme celui de Déméter et de Dionysos, de se rapprocher au point peut-être de proposer une fusion des pratiques voire des traditions rituelles. Cela représente une autre possibilité de partage et de diffusion de spécificités rituelles qui vient s'ajouter à celles déjà définies, soit l'emprunt à un imaginaire largement diffusé, la reprise d'un vocabulaire fonctionnel de base issu des cultes publics (prêtre, hiérophante, épimélète...), l'adoption d'une 'invention' rituelle d'un groupement extérieur (ßoukóloı), ou encore la création de fonctions uniques et spécifiques pour les besoins particuliers d'un groupement précis, que ce soient des besoins proprement rituels ou plus largement honorifiques, l'un n'excluant pas l'autre naturellement.

\section{Du langage associatif aux communautés chrétiennes : Ignace d'Antioche}

Cette association regroupant des Démétriastes et des mystes de Dionysos, ouvre une autre perspective sur les moteurs des échanges, sur la dynamique des transmissions, et sur leur rapport précis et direct avec les rituels : il convient en effet de se demander si la diffusion à large échelle d'un vocabulaire mystérique « unifié » n'a pas favorisé ce rapprochement. On sait en effet que le terme de myste a connu un développement géographique fulgurant dès le $\mathrm{I}^{\text {er }}$ siècle de notre ère en Asie Mineure et sur la rive gauche du Pont-Euxin notamment; cette propagation comme spontanée et contagieuse ne s'est pas faite sans une certaine banalisation du terme et donc de son référent cultuel ${ }^{49}$. Et l'on aurait tort d'en sous-estimer la composante sociale, élitaire, dirigée dans le sens d'une hiérarchisation de prestige des rites - et avant tout de leur dénomination -, comme de ceux qui les partagent et les promeuvent ${ }^{50}$. Si les termes s'éloignent donc d'une 
expérience rituelle précise et cernable, nous n'en assistons pas moins à l'établissement et à la diffusion d'une koinè mystérique - par le biais du vocabulaire tout au moins.

41 Cette constatation nous conduit à la prochaine étape de cette enquête, celle qui touche la reprise d'un vocabulaire et peut-être d'un savoir religieux par les auteurs chrétiens. Ce que nous appelons une koinè mystérique, comprise comme la large diffusion de quelques termes spécifiques (mystes, $\mu \nu \sigma \tau \eta ́ p i \alpha$, hiérophante), et comme une globalisation de spécificités rituelles derrière un vocabulaire uniforme ${ }^{51}$, a eu un impact sur les premiers auteurs chrétiens : de nombreuses études ont montré qu'à partir de la fin du $\mathrm{II}^{\mathrm{e}}$ siècle de notre ère, la terminologie des cultes à mystères est devenue courante pour définir la liturgie et la pratique rituelle des sectateurs de Jésus ${ }^{52}$. Des termes comme $\mu \nu \sigma \tau \eta ́ p i \alpha$ et, dans une moindre mesure, $\tau \varepsilon \lambda \varepsilon \tau \eta ́$, ou bien encore l'adjectif $\mu \nu \sigma \tau 1 \kappa o ́ \varsigma$, se sont diffusés dans les ouvrages chrétiens; ils définissent non seulement l'ensemble des rites ou des doctrines des sectateurs de Jésus, mais aussi des formes de culte plus spécifiques comme le baptême et l'eucharistie ${ }^{53}$. Le recours à des termes issus du vocabulaire mystérique par les auteurs chrétiens a-t-il été voulu pour créer une connivence, un réflexe culturel autant que cultuel autour des pratiques mystériques de façon à faire comprendre la foi nouvelle sur les bases d'un savoir communément partagé ? Ignace d'Antioche, l'un des premiers auteurs chrétiens dont nous avons connaissance, qui voyagea dans plusieurs cités d'Asie Mineure au début du II $^{\mathrm{e}}$ siècle de notre ère représente une base d'analyse privilégiée dans ce contexte.

42 Évêque de la ville d'Antioche entre 110 et 120 de notre ère, sous Trajan et Hadrien, Ignace fut arrêté par les autorités impériales et condamné au supplice dans l'amphithéâtre de Rome: nous ne connaissons pas les raisons qui ont conduit à cette condamnation, s'il s'agissait d'une persécution locale contre les chrétiens ou d'un conflit interne à la communauté chrétienne d'Antioche. Sur son chemin vers la capitale, Ignace s'arrêta dans plusieurs villes d'Asie Mineure et envoya des lettres aux communautés chrétiennes d'Éphèse, Magnésie, Tralles, Philadelphie, Smyrne et Rome ${ }^{54}$. Selon la plupart des chercheurs, ces lettres datent du début $d u{ }{ }^{\mathrm{e}}$ siècle de notre ère, plus précisément entre les années 110-120, soit au moins un demi-siècle avant que l'usage des termes mystériques ne devienne courant dans les textes chrétiens ${ }^{55}$.

43 C'est pendant son séjour à Smyrne qu'Ignace rédige la Lettre aux Éphésiens, à la suite de la visite de l'évêque d'Éphèse, Onésime, et de la délégation éphésienne. Ignace montre connaître les problèmes qui agitaient les sectateurs de Jésus dans la grande ville d'Artémis, les divisions et les conflits internes à l'église, et il exhorte les fidèles à se soumettre à l'autorité de l'évêque et à se garder des « chiens enragés » ( qui viennent d'ailleurs et éloignent les fidèles de la vérité ${ }^{56}$. Avant d'entrer dans le corps de la Lettre aux Éphésiens, il convient de rappeler que les communautés chrétiennes dont nous parle Ignace sont des petites communautés : dans une grande ville comme Éphèse, les chercheurs ont avancé l'hypothèse d'une centaine de chrétiens ${ }^{57}$.

Les Lettres d'Ignace représentent, avec la Didachè et la Lettre de Clément aux Corinthiens, les premiers témoignages de la littérature chrétienne. Nous sommes là, donc, en pleine formation de l'identité (ou des identités) chrétienne(s), à un moment où le lexique chrétien est encore en voie de construction. Il suffit de rappeler qu'Ignace est l'un des

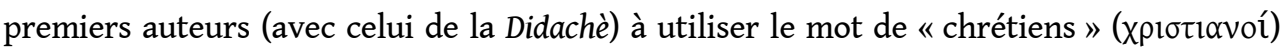
pour définir les sectateurs de Jésus, un terme qui n'était pas présent dans les lettres de Paul $^{58}$. Ignace est également le premier à utiliser l'expression «église catholique» 


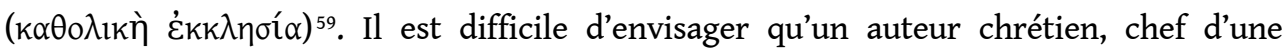
communauté religieuse, qui voyage dans les villes d'Asie Mineure ne se trouve pas confronté, de manière directe ou indirecte, au monde des associations si profondément ancré dans les cités grecques. Le vocabulaire utilisé par Ignace met en évidence cette connaissance du monde cultuel d'Asie Mineure, comme l'étude de Philippe Harland l'a clairement montré ${ }^{60}$. On pourrait difficilement en effet attribuer la connaissance du langage associatif à la formation scolaire ou à la paideia de l'auteur chrétien, car il s'agit de termes qui appartiennent spécifiquement à un contexte cultuel.

Mais venons-en directement au texte de la Lettre aux Éphésiens et aux références au monde associatif grec de l'époque. Le premier souci d'Ignace est l'unité de l'église, de la communauté autour de son évêque, une unité qui sous-entend à la fois cohérence

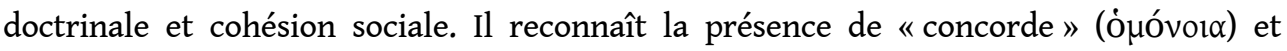

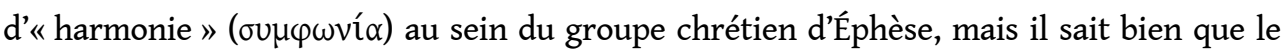
conflit et la désagrégation sont toujours aux aguets. C'est pourquoi il adresse aux Éphésiens le souhait qu'ils puissent devenir un «chœur» (xopóc) qui chante à

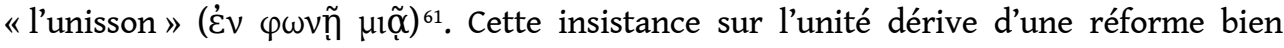
précise qu'Ignace a introduite dans les communautés chrétiennes de l'époque. À la différence de Paul, l'évêque d'Antioche propose - et c'est le premier à le faire - une église organisée d'un point de vue hiérarchique, avec trois niveaux et trois fonctions : 1 . l'évêque ; 2 . les presbytres ; 3 . les diacres ${ }^{62}$. Cette « réforme » d'Ignace déclencha plusieurs réactions négatives et les conflits internes aux églises qui transparaissent dans les lettres dont nous disposons sont le résultat du nouveau rôle de l'évêque dans la communauté chrétienne.

46 C'est dans cette perspective que nous pouvons lire l'exhortation d'Ignace, adressée aux Éphésiens, à former une procession qui fait écho aux associations :

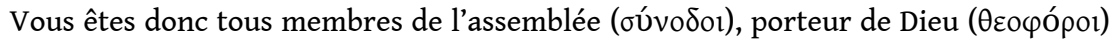

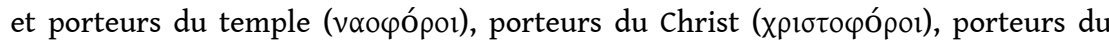

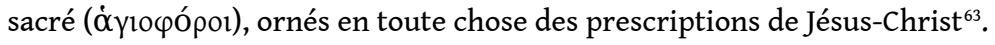

47 Ignace imagine une procession chrétienne dont les fonctions sont calquées sur les associations qui existaient dans les cités grecques d'Asie Mineure : en effet, les substantifs en -phoros étaient très courants dans le vocabulaire associatif, tout comme dans les processions civiques qui connaissaient ce type d'agents rituels. Mais les références maniées par Ignace ne sauraient se satisfaire de ce premier niveau de lecture. La religion des chrétiens se configure, dans son texte, comme un culte à mystères : l'auteur la définit explicitement comme $\mu \nu \sigma \tau \eta ́ p ı \alpha$, employant justement le pluriel répandu dans les cultes à mystères des mondes grec et romain et non, comme Paul, le singulier $\mu v \sigma \tau$ ṕpiov ${ }^{64}$. Ignace joue clairement sur les mots et sur les concepts mystériques. Le parallélisme avec les associations mystériques est d'autant plus frappant que l'auteur utilise également le terme $\sigma u \mu \mu u ́ \sigma \tau \alpha \iota ~ \Pi \alpha u ́ \lambda o v$ pour définir les chrétiens :

Vous êtes le passage ( $\left.\pi \alpha \alpha_{\rho} \rho \delta \circ \zeta\right)$ pour ceux qui sont morts pour Dieu, vous êtes les

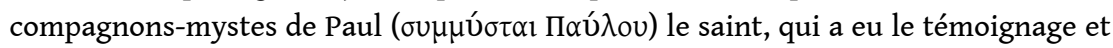
est digne d'être appelé bienheureux ${ }^{65}$.

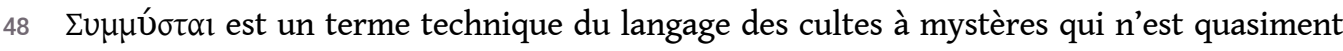
pas attesté dans les sources littéraires ${ }^{66}$. En outre, ce terme renvoie de façon particulière à la sphère rituelle dionysiaque : non seulement on le trouve attesté, à plusieurs reprises, dans les inscriptions des associations dionysiaques ${ }^{67}$, mais encore et surtout, sur le plan plus profond des concepts rituels, c'est à travers cette notion réciproque que l'on 
construit et exprime un rapport fort et à double sens, entre les membres de la communauté d'une part et entre le dévot et son dieu de l'autre. Car l'expérience rituelle bachique et le mode de présence du dieu face à ceux qui l'honorent donnent tout son sens, son double sens, au préfixe $\sigma u \mu-;$ par le fait que le dieu a été, lui aussi, initié et à ses propres mystères, il est le compagnon d'initiation des mystes humains, comme ceux-ci le sont du dieu et comme ils le sont ensemble au sein de l'association qui les rassemble. Ce rapport particulier établi entre la divinité et les hommes, par le partage du rite d'initiation, pouvait d'autre part évoquer, dans une optique chrétienne, le lien particulier entre les chrétiens et Jésus, par le partage du baptême, tout autant que renvoyer à la communauté des hommes, unis par une même expérience rituelle et suivant l'exemple de Paul, le converti ${ }^{68}$.

49 Si cette utilisation du concept de $\sigma u \mu \mu u ́ \sigma \tau \alpha$ par Ignace peut évoquer une expérience initiatique et communautaire particulière, teintée de références dionysiaques, un second registre référentiel lie le vocabulaire d'Ignace à la sphère du dieu : le recours au langage théâtral. On a déjà rappelé l'exhortation à former un chœur adressée aux chrétiens

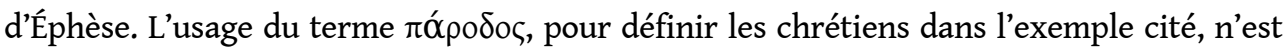
sans doute pas innocent non plus. De plus, juste avant le passage évoquant la procession

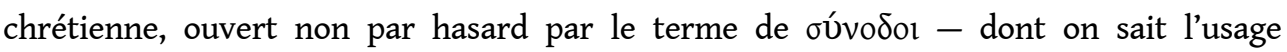
'œcuménique' pour désigner les guildes d'acteurs professionnels -, Ignace utilise une métaphore liée aux mises en scène du théâtre : il affirme que les chrétiens sont

les pierres du temple du Père, préparés pour la construction de Dieu le Père, soulevés en haut par la grue ( Saint Esprit comme un câble ${ }^{69}$.

50 En faisant de Jésus la $\mu \eta \chi \alpha v \eta ́$, la grue, indispensable à la construction de l'Église chrétienne, Ignace ne pense-t-il qu'à une simple métaphore du domaine de la construction? N'a-t-il pas en vue, avec la $\mu \eta \chi \alpha v \eta ́$, l'outil théâtral qui permettait de déplacer les acteurs en les faisant monter au-dessus de la skènè, de leur faire ainsi dépasser leur limites humaines comme il siérait dans une métaphore appliquée à la construction de l'Église chrétienne ? Cela reste hypothétique. Mais on notera que dans la littérature chrétienne en langue grecque, le langage métaphorique du théâtre sera bien présent, quel que soit le discrédit jeté sur les spectacles : Clément d'Alexandrie, dans son Protreptique aux Hellènes, après avoir pourtant condamné les concours des Lénées, qui sont le lieu de l'ivresse et de la folie en l'honneur de Dionysos, ne renonce pas pour autant à la thématique du théâtre. C'est qu'il ne veut pas laisser aux seuls païens la possibilité d'exploiter les ressources métaphoriques et allégoriques des spectacles ${ }^{70}$. Aux concours du monde gréco-romain le texte oppose alors de nouveaux concours chrétiens, parce que le Christ est « le Logos céleste, qui, dans le véritable concours, reçoit la couronne sur la

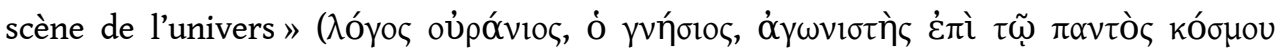

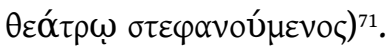

51 Étant donné qu'Ignace n'avait pas d'exempla littéraires chrétiens à suivre dans cette reprise métaphorique et que d'autre part nous ignorons sa formation scolaire et son éventuelle connaissance des textes classiques qui reprenaient ce type de langage, il est tout à fait envisageable que cet usage vienne d'ailleurs, des pratiques associatives et mystériques en général et, en particulier, du monde dionysiaque d'Asie Mineure. Ignace était à Smyrne, lors de la rédaction de la Lettre aux Éphésiens, cité où les inscriptions témoignent de l'existence d'associations dionysiaques au sein desquelles initiés et acteurs cohabitaient $^{72}$. Nous pourrions avancer l'hypothèse que la présence de l'aspect théâtral et 
de l'aspect mystérique dans le texte d'Ignace dérive aussi de l'observation directe d'une expérience associative qu'il pouvait connaître ${ }^{73}$.

Un certain 'savoir partagé' dont nous voyons les racines notamment dans les associations dionysiaques devient le modèle à travers lequel construire la représentation de la communauté chrétienne. Nous avons, chez Ignace, tout d'abord un besoin de constituer un vocabulaire, une nécessité linguistique qui trouvait réponse dans les formes cultuelles que l'auteur avait connues à la fois directement - au cours de son voyage vers Rome - et indirectement - grâce aux témoignages des délégations qui lui avaient rendu visite. Mais la récupération du langage associatif trouve aussi une autre explication, interne aux problèmes des communautés chrétiennes de la première moitié du $\mathrm{II}^{\mathrm{e}}$ siècle de notre ère. Nous avons fait référence à la réforme des églises proposée par Ignace et au rôle nouveau que l'auteur chrétien souhaite donner à l'évêque. Dans cette perspective, représenter les chrétiens selon le modèle associatif, en les définissant par les termes de $\sigma u \mu \mu v ́ \sigma \tau \alpha$ l,

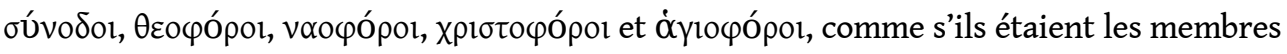
d'une association dotée de ses charges et ses titres, pourrait être interprété aussi comme un moyen de promouvoir sa propre réforme des églises. Ignace s'inspire du modèle des associations qui existaient dans le monde des cités d'Asie Mineure et, sans doute notamment du modèle dionysiaque, afin de transmettre implicitement une image structurée et hiérarchisée, telle que celle qu'il cherchait à imposer au sein des communautés chrétiennes. L'opération d'Ignace sur le langage associatif dévoile un aspect de la formation de l'identité chrétienne qui essaie de s'enraciner dans le vocabulaire local des cités d'Asie Mineure, d'autant plus que la lecture de ses Lettres révèle des situations locales différentes les unes des autres ${ }^{74}$. Les termes rituels relevant du contexte initiatique choisis par un auteur chrétien comme Ignace d'Antioche pour s'adresser à la communauté de sectateurs de Jésus à Éphèse témoignent ainsi d'une complicité basée sur un 'savoir religieux' partagé, un savoir qui se manifeste comme la reprise d'un substrat général répandu dans les cités d'Asie Mineure et plus particulièrement à Éphèse. Ce phénomène de reprise induit une certaine perméabilité, au moins métaphorique, du vocabulaire cultuel laissant deviner une empreinte culturelle à large échelle, base d'un consensus utilisable pour faire entendre un message nouveau.

\section{Le hiérophante public d'Éphèse ou la reconnaissance d'un spécialiste en compétences rituelles}

Les deux dossiers convoqués jusqu'ici nous ont permis d'aborder, par un biais forcément partiel mais concret, la question de la transmission des savoirs ou des références en matière cultuelle à des échelles différentes et dans des contextes bien distincts. Les associations dionysiaques posent la question des référents à l'œuvre dans la création d'un culte pensé comme ciment d'une communauté de peu d'ampleur, dont le rôle public ou officiel n'est pas le principal moteur mais dont les contours généraux entrent en résonnance consensuelle avec les coutumes rituelles de la société dans laquelle ces microcosmes prennent pied. Si l'on ne peut parler d'officialité lorsqu'il est question d'une congrégation chrétienne du début du II $^{\mathrm{e}}$ siècle, les propos d'Ignace, bien qu'adressés aux Éphésiens, ont comme objectif l'organisation d'une structure qui dépasse le cadre local et vise l'universalité, bien que s'inscrivant à contre-courant des traditions cultuelles reconnues publiquement, ou peut-être à cause de cette non conformité au modèle ambiant. Un dernier dossier permettra d'ouvrir la réflexion sur la reconnaissance et la 
transmission de compétences rituelles au sein même de la pratique des cultes publics et officiels de la cité d'Éphèse, autre volet, parfaitement complémentaire, de la question.

Il s'agit d'une inscription éphésienne $d u{ }{ }^{e}{ }^{e}$ siècle de notre ère, un règlement « sacrificiel $»^{75}$ dont le contenu pourrait correspondre à une réalité rituelle plus ancienne si l'on en croit l'intitulé même du texte annoncé comme « le résumé de la loi ancestrale »

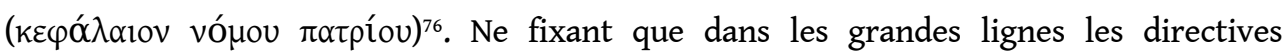
proprement rituelles, le texte rappelle globalement les devoirs sacrés du prytane, le

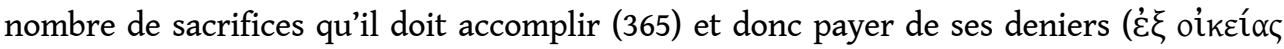
$\delta v v \alpha ́$ $\mu \varepsilon \omega \varsigma$ 1. 9-10). Suit alors la mention qui retiendra notre attention ici (1. 10-12) :

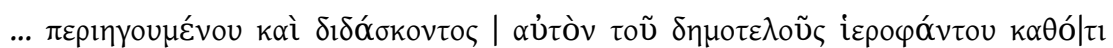

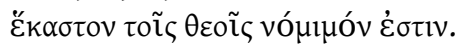

le hiérophante public lui donnant les explications et l'enseignement sur la manière

de s'y prendre selon ce qui est, dans chaque cas, la tradition pour les dieux.

Cette précision est capitale : nous y voyons clairement apparaitre la séparation des fonctions entre celui qui fait et celui qui sait et sait faire, en matière rituelle. Le prytane, nouveau chaque année, accomplit le devoir religieux que sa charge implique, au nom de la cité, sans maitriser la complexité des coutumes à observer dans chaque cas de figure, selon le dieu et l'occasion. Le savoir rituel est détenu par le hiérophante public : par sa permanence, il assure la transmission des vó l'autre. Et ce savoir se transmet par des explications - périégèse - une façon de « faire le tour » de la question, en expliquant mais aussi sans doute en transmettant les récits, les formules, le hieros logos qui sous-tend le rite, particulier à chaque occasion. À cette

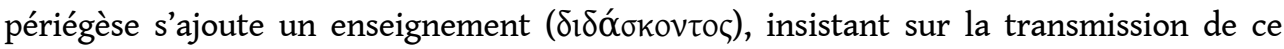
savoir rituel, sur la formation théorique et pratique à laquelle doit se soumettre le prytane, sous la direction du hiérophante, pour effectuer sans faux pas les rites qu'exige sa fonction de représentant de la cité. Cette fonction et cette autorité reconnue du hiérophante sont soulignées une seconde fois dans la même inscription, au moment d'évoquer la répartition des $\gamma \varepsilon ́ p \alpha$ entre les différents officiants ou dignitaires (1. 18-22).

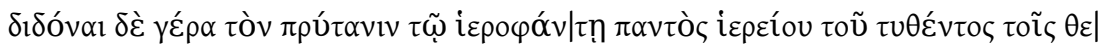

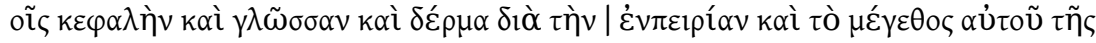

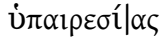
Que le prytane donne au hiérophante lors de tout sacrifice offert aux dieux la tête, la langue et la peau à cause de son expérience et de la grandeur de son service.

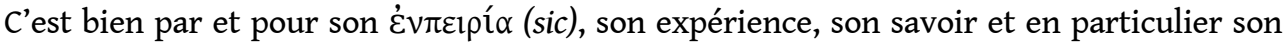
savoir-faire que le hiérophante est gratifié, pour chaque victime sacrifiée, de parts de choix (tête, langue, peau). La reconnaissance de son savoir fondamental au bon déroulement des rites officiels s'exprime à la fois verbalement et matériellement en termes de parts dévolues. Nous voyons ainsi de l'intérieur le fonctionnement religieux d'une cité, Éphèse en l'occurrence. Maîtrise rituelle et fonction représentative sont distinctes, mais parfaitement complémentaires, pour assurer le salut de la communauté dans son ensemble. Cet état de fait reflète-t-il une réalité fonctionnelle valable au-delà du cas particulier d'Éphèse ? C'est ce que laisse supposer un passage de Denys d'Halicarnasse

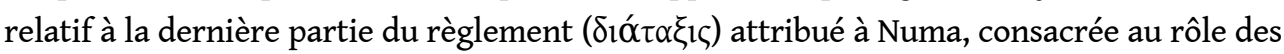
pontifices à Rome ${ }^{77}$ :

Ils [les pontifices] établissent des règles pour tous les rites qui, n'étant pas encore fixés par écrit ni consacrés par l'usage, leur semblent réclamer l'appui de lois et de coutumes. Ils contrôlent les fonctions de tous les magistrats auxquels incombe un sacrifice ou un service du culte, et celles de tous les prêtres. Ils veillent à ce que les 
serviteurs et les ministres qu'ils utilisent pour l'accomplissement des rites ne commettent aucune faute à l'égard des lois sacrées. [...] Par conséquent, qu'on veuille les appeler hierodidaskaloi, hieronomoi, hierophylakes ou - ce qui nous semble préférable - hierophantai, on ne s'écartera pas de la vérité ${ }^{78}$.

Le domaine d'action et de compétences reconnu aux pontifes romains, tel qu'il est ici traduit en termes et concepts grecs, se situe bel et bien entre l'enseignement, l'édification des lois rituelles ou leur conservation, ou encore la surveillance de l'application pratique des préceptes rituels - une amplitude fonctionnelle vaste, mais cohérente. Si les trois premiers termes envisagés pour 'traduire' cette vaste fonctionnalité, renvoient à des fonctions explicites - l'enseignement (i $\varepsilon \rho \circ \delta 1 \delta \alpha ́ \sigma \kappa \alpha \lambda o 1)$, la mise sur pied des lois

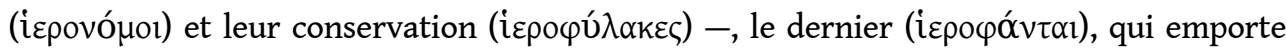
l'adhésion de Denys et dont il se servira couramment pour désigner les pontifices, est plus général, plus englobant, renvoyant à l'expression ou à l'explication des choses sacrées. Comme dans le texte de l'inscription éphésienne, nous voyons à l'œuvre la complémentarité parfaite entre compétence rituelle et représentativité officielle, qui suit le principe de milice, soit la non professionnalisation des tâches effectuées au nom et pour l'État, très généralement de mise tant dans les cités grecques qu'à Rome. L'intervention d'un spécialiste des coutumes religieuses, du savoir et savoir-faire rituels permet et garantit la tenue des sacrifices 'dans les normes' respectant les particularités propres à chaque occasion, à chaque divinité, à chaque type de sacrifice et formant le pont de tradition par-delà la succession des magistrats inexpérimentés en la matière. C'est bien cette idée de pont que reprend Denys, comme d'autres, comme explication étymologique du terme de pontifex; idée suffisamment forte pour qu'il l'exprime en tête

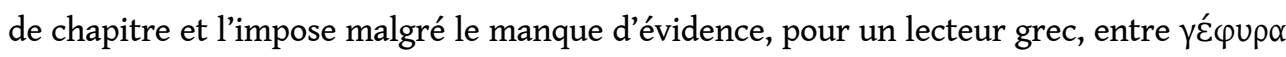
grec et pons latin. Le lien et le poids de cette explication sont donc plus dans le concept que dans le terme. Et si Denys s'en tient dans le texte à «l'entretien du pont de bois » (l'archaïque pont Sublicius) pour ancrer cette étymologie, rien n'empêche de penser qu'il songe plus largement à l'idée de transmission de la tradition, de pont de rites et de coutumes, que la suite du texte développe de manière flagrante.

L'inscription éphésienne consacrant les compétences rituelles du hiérophante et sa mise en parallèle avec le texte de Denys permettent-elles d'envisager la présence d'un spécialiste de la transmission rituelle dans d'autres cités qu'Éphèse ? En d'autres termes, avons-nous avec cette inscription éphésienne un cas particulier ou au contraire l'expression unique dans notre documentation d'un phénomène généralisé non exprimé usuellement? L'hésitation de Denys sur le terme approprié, au moment de traduire, non le mot mais l'amplitude de fonctions des pontifices, souligne bien l'absence de parallèle fonctionnel direct et complet dans la tradition grecque. Le morcellement, dans le monde grec, des traditions religieuses en autant d'entités distinctes que de cités y est sans doute pour quelque chose. Si la question mérite d'être posée, l'absence de témoignages antiques sur le sujet empêche tout développement fondé et la chaîne d'interrogations suscitées par cette aporie nous entraînerait trop loin et sur un terrain trop incertain ${ }^{79}$. 


\section{Du hiérophante public d'Éphèse à la lettre d'Ignace d'Antioche : savoirs partagés entre référence cultuelle et culturelle} hiérophante dans la transmission des bonnes coutumes en matière de pratique rituelle nous servira de référent, d'horizon réflexif et de faire-valoir, en creux ou en relief, des quelques cas de figure rencontrés dans ce parcours. La séparation claire opérée, dans les rites publics, entre la compétence rituelle du hiérophante et la représentativité du prytane nous conduit en effet à quelques réflexions sur les modalités de fonctionnement d'une communauté cultuelle. Nous remarquons que la transmission d'un savoir et savoirfaire religieux spécifique est confiée à une personne, et non à des documents écrits, « lois sacrées » ou autres, auxquels on renverrait. Il est d'ailleurs significatif de constater que le « règlement sacrificiel » qui précise ces dispositions, document écrit de référence, ne contient justement pas les prescriptions rituelles, mais seulement la répartition des tâches. Le savoir religieux, rituel, se transmet par l'oral, non par l'écrit. L'autorité en matière religieuse est une personne à laquelle on reconnaît un savoir, une connaissance des traditions, non un texte, une loi fixée par l'écrit ${ }^{80}$. On peut y voir plusieurs raisons. Outre la multiplicité des situations à expliciter qui déboucherait immanquablement sur un document de référence extrêmement volumineux et complexe, on retiendra deux aspects. La référence à une personne insiste sur le côté humain des rites. L'autorité religieuse vient d'abord de celui qui connaît les rites, non d'un référent divin direct. Si l'oracle est consulté, et peut ainsi faire office de référent divin direct, ce sera avant tout pour légitimer des rites proposés par les hommes, auxquels le dieu donnera ou non son aval. D'autre part, le savoir nécessaire à la tenue 'sans bavure' des rites est avant tout un savoir-faire, une pratique, le bon geste, la bonne gestion de l'espace, la bonne temporalité dans le rite, la bonne formule prononcée en temps requis - autant de dimensions qui échappent à toute mise par écrit et à toute diffusion en dehors de l'expérience pratique, visuelle et même émotionnelle, ou sensorielle.

61 Le hiérophante transmet donc, par-delà la succession des prytanes annuels, une pratique fiable. Il est bien ainsi un passeur de tradition, un bâtisseur de ponts, une garantie pour la communauté dans son ensemble que les rites seront efficaces et lui assureront sa sauvegarde. Nous ne sommes ainsi pas très loin du modèle théorique et fictionnel de l'oracle de Magnésie, avec la nécessité de recourir à une autorité reconnue pour asseoir un culte nouveau, dont l'oubli menace la communauté. Dans le cas de Magnésie, la légitimité viendra certes non du dedans mais du dehors, avec la référence au berceau thébain et Cadméen du culte dionysiaque. Mais le processus fictionnel de Magnésie est en 
parfaite adéquation avec la procédure bien réelle d'Éphèse ; c'est à des personnes et non à des règlements qu'est confiée la transmission du savoir et du savoir-faire nécessaires. Au travers de ces deux cas de figure, c'est bien la même conception qui est exprimée, celle la transmission de génération en génération - donc à dimension humaine - de traditions 'sanctifiées' par le temps et l'origine ancestrale: c'est ce que nous appelons une transmission verticale des savoirs.

Mais dans un même temps, ce recours à la tradition, comme clef de légitimation, repose justement, par sa dimension humaine et orale, sur le discours. L'oralité de la transmission permet une double manœuvre : elle ouvre la voie à une certaine plasticité des rituels et à leur adaptation en douceur à l'évolution de la société et de ses besoins, et elle articule à ces potentielles nouveautés un discours légitimant basé sur le recours à la tradition, orale et bien humaine, autre manière de bâtir des ponts ou du moins de les entretenir ${ }^{81}$.

L'étude du dossier restreint des associations dionysiaques éphésiennes n'offre pas de parallèle à cette transmission verticale des savoirs ou savoir-faire. La nature des documents exploités et le seul recours possible aux titres de fonctions rituelles sont peutêtre des raisons à cette absence; et l'on peut se demander également dans quelle mesure le caractère non officiel des rites associatifs, le fait qu'ils ne soient pas effectués à proprement parler pour la communauté entière, ne rendent pas inutile ou moins nécessaire le recours à une autorité reconnue et légitimante. Cette séparation de rites entre sphère 'publique' et 'privée' qui s'impose à nos esprits modernes, risque toutefois d'occulter une réalité bien plus perméable dans la réalité antique. Combien d'associations ne sont-elles en effet pas mises à contribution et intégrées dans les pratiques 'officielles' des rites des diverses cités ${ }^{82}$ ? Combien ne choisissent-elles pas d'intégrer le modèle d'autoreprésentation de la cités ${ }^{3}$ ? La bonne marche d'une cité ne se mesure pas ainsi à l'aune de l'orthopraxie des seuls rites officiels mais englobe toutes les manifestations rituelles de quelque envergure et visibilité publiques directes qu'elles soient. L'exemple théorique donné par l'oracle rendu aux Magnètes atteste bien la complémentarité du culte public, représenté par la construction d'un temple et l'installation d'un prêtre, et des thiases, établis par les trois Ménades importées de Thèbes et fonctionnant en microcosmes indépendants.

Quoi qu'il en soit, et si cette transmission d'une tradition 'verticale' n'est pas perceptible dans les documents associatifs analysés, c'est à une autre dimension de la transmission que nous rend attentifs le dossier éphésien. Devant la nécessité et en même temps la liberté de créer un culte, point focal et rassembleur de ces microcosmes sociétaux, les différents dionysiastes éphésiens ont opté pour des solutions variées et complémentaires: innovations créées pour répondre aux besoins précis de tel ou tel groupe, emprunts d'un groupe à un autre, d'une ville à une autre ou fusions de rites entre communautés vouées à des divinités différentes, sans négliger l'utilisation d'un vocabulaire globalisé formant une koinè, que ce soit au niveau des fonctions rituelles de base et "neutres », comme celle de prêtre, ou sur le plan de l'adéquation au vocabulaire mystérique "à la mode ». L'ensemble de ces démarches s'apparente davantage à ce que nous appelons une diffusion horizontale des savoirs ; laquelle repose sur des motivations variées, comme la concurrence, entre associations dionysiaques d'une même ville ou de villes rivales, le prestige de tels ou tels titres, la recherche de hiérarchisation des élites associatives ou encore les besoins véritablement rituels d'une fonction liturgique précise en adéquation avec l'orientation cultuelle choisie par le groupement. Cette diffusion s'opère sans recours à une quelconque profondeur temporelle et traditionnelle comme 
justificatif, mais repose simplement sur un savoir contemporain, accessible à tout un chacun et largement diffusé.

C'est cette circulation horizontale et synchronique dont rend compte encore Ignace d'Antioche, prouvant par là même la publicité, dans son sens premier, des manifestations associatives et des référents qui servent de socle aux rituels des uns comme des autres. C'est sur cette connaissance partagée de certaines réalités rituelles largement diffusées que l'auteur chrétien ancrera un discours neuf, entrant dans le langage commun pour exprimer des liens nouveaux autant entre adeptes chrétiens qu'entre hommes et Dieu, autre dialogue entre horizontalité et verticalité.

Doit-on dès lors considérer cet usage chrétien du vocabulaire et des concepts associatifs comme une référence cultuelle ou culturelle ? La question se pose dans les mêmes termes pour ce qui est des transmissions 'horizontales' au sein des associations. La publicité des rites ou du moins de certains d'entre eux, brise d'elle-même cette catégorisation moderne. En étant largement connus de tous, certains rites passent automatiquement du cultuel au culturel. Le rite est une référence culturelle dans son essence, un point de consensus dont l'oralité de la transmission garantit l'adaptation harmonieuse aux besoins toujours actuels. Les seules références que l'on pourrait qualifier de véritablement culturelles, celles qui font allusion aux récits concernant l'installation de Dionysos et de son culte, à Thèbes comme matrice cultuelle, ou aux grands poncifs bachiques dont sont nourris tant les textes que les images (thyrse, nébride etc.), se révèlent très marginales et ne font pas recette dans les fonctions rituelles. Doit-on s'en étonner? La dimension événementielle du savoir religieux, fondée sur un savoir-faire pratique n'a que faire en fin de compte de références « culturelles »; dans un savoir religieux en action, c'est le rite effectif qui devient lui-même référence culturelle.

\section{NOTES}

*. Une première version de cette étude a été présentée en septembre 2012 dans le cadre du $6^{\mathrm{e}}$ atelier Chicago-Paris sur les religions anciennes, consacré au thème «Les savoirs religieux Religious Knowledge» (24-25 septembre 2012 INHA, Paris). Que les organisateurs trouvent ici l'expression de notre reconnaissance. Francesco Massa a réalisé ce travail dans le cadre du laboratoire d'excellente LabexMed portant la référence 10-LABX-0090 et a bénéficié d'une aide de l'État français gérée par l'ANR au titre du projet investissements d'Avenir A*MIDEX portant la référence $\mathrm{n}^{\circ}$ ANR-11-IDEX-0001-02.

1. STAVRIANOPOULOU (2006 a et b), BRULÉ (2009), HEKSTER, SCHMIDT-HOFNER, WITSCHEL (2009), CABOURET, CHARLES-LAFORGE (2010), SCHEID (2010), CHANIOTIS (2011), BRICAULT, BONNET (2013 a), BELAYCHE (2013). Les études concernant le rôle de l'écriture dans le fonctionnement des pratiques rituelles des mondes grec et romain éclairent elles aussi la question, voir GORDON (1990), SCHEID (1994), NORTH (1998) HENRICHS (2003), BELAYCHE, MASSA (2013). Sur le concept de «mutations religieuses»cf. PIRENNE-DELFORGE, SCHEID (2013) avec la nécessaire distinction entre degré et nature de la différence (p.313) et la reconnaissance de changements qui affectent le religieux parce qu'ils sont simplement le reflet d'un « changement culturel général» (p.312); sur l'historiographie de ces «mutations religieuses », voir BRICAULT, BONNET (2013 b). 
2. JACCOTTET (2003), I, p. 54-58, 200-201 et passim, JACCOTTET (2006).

3. Par exemple KeRn (1900) $\mathrm{n}^{\circ} 215$; MERKELBACH, STAUBER (1998), I, $\mathrm{n}^{\circ} 02 / 01 / 02$, p. 187-191, JACCOTTET (2003), II, nº 146, p. 244-247, JACCOTTET (2005), p. 192-193.

4. L'oracle a-t-il été effectivement rendu par Delphes ou n'est-il qu'une fiction, une composition de circonstance visant à soutenir la politique magnète à un moment précis de son histoire? POMTOW (1896), part. p. 754-756 a été le premier à voir l'oracle comme un faux et à proposer une lecture des circonstances historiques qui expliquent le recours fictif à l'autorité delphique (demande d'asylie de Magnésie), plaçant cette inscription sur le même pied que le texte connu

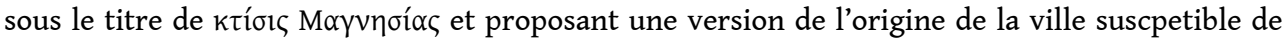
soutenir la demande d'asylie de la cité (I. Magn. 17, FGrH 482 F 3). HENRICHS (1978), p. 124-130 en revanche plaide pour l'authenticité du texte et donc de l'oracle qu'il situe, sur des critères linguistiques et historiques entre 278 et 250 av. notre ère, alors que le texte de l'inscription originale (copiée par Apollonios au II ${ }^{\mathrm{e}}$ siècle de notre ère) serait postérieur à 207/6 av. notre ère. Pour notre part, nous nous rallions aux arguments mettant en doute l'authenticité de l'oracle ; la datation du texte à la fin $\mathrm{du}_{\mathrm{III}}^{\mathrm{e}}$ siècle avant notre ère pour l'inscription copiée par Apollonios à l'époque d'Hadrien peut être maintenue sans pour autant induire une quelconque authenticité de l'oracle lui-même. Pour un aperçu historiographique des commentaires suscités par l'inscription, cf. HENRICHS (1978), p. 124-125, n. 7. Plus récemment, cf. KNOEPFLER (1989), p. 57 et passim, JACСоTтET (2003), I, p. 77-78, 134, II, p. 244-247.

5. On notera que le texte de l'oracle ne mentionne pas le nombre de femmes concernées. C'est la suite de l'inscription, qui manifeste l'obéissance des délégués aux prescriptions apolliniennes, qui spécifie que trois ménades ont été prises à Thèbes. Chiffre significatif s'il en est dans cette tradition thébaine du culte dionysiaque.

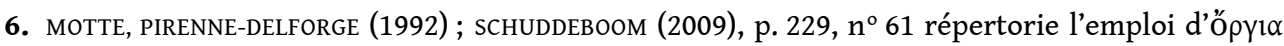
dans cette inscription sous sa rubrique "Dionysiac rites" (p. 227-230) et non sous celle de "Cult objects" (p. 237), qui ne contient que l'épigramme funéraire de la prêtresse Alcméonis de Milet ( PEEK (1980) 1344 ; MERKELBACH, STAUBER (1998) $\mathrm{n}^{\circ} 01 / 20 / 21$, p. 138, JACCOTTET (2003), II, $\mathrm{n}^{\circ} 149$, p. 250-251, I, p. 74-77, 133-134). La présence d'un verbe tel que "porter", comme dans le cas de cette épigramme, rend manifeste le sens concret et matériel des ópүı ; on aurait tort pour autant, croyons-nous, de restreindre ce sens matériel aux seules occurrences parfaitement explicites de par leur contexte syntaxique. L'hypothèse d'une complémentarité, dans l'oracle de Magnésie, entre les vó $\mu \iota \alpha$ et les ópүı $\alpha$, entre une manière de faire et/ou de dire et des objets nécessaires au bon déroulement des rites nous paraît au moins envisageable.

7. Dans l'optique soutenue par le texte, la notion de «terre sainte » essaie de rendre l'absolu référentiel invoqué par l'entremise de Thèbes.

8. Cf. supra note 4 .

9. Sur les rapports entre textes littéraires et pratiques rituelles dionysiaques voir HENRICHS (1978), p. 155-156 et JAсCоттЕт (1998) et (2003), I, p. 92-93, 139-140.

10. Sur le succès des Bacchantes d'Euripide aux époques suivantes on se référera aux introductions au texte tragique de ROUX (1970), I, p. 72-77 et de SEAFORD (1996), p. 52-54. Voir aussi MASSA (2010).

11. Théocrite, Idylles, 26, 1-2.

12. Cf. Théocrite, Idylles, 26, 9. Sur cette idylle voir GRONINGEN (1963) et CUSSET (1997).

13. Sur le rôle des femmes dans les pratiques rituelles dionysiaques voir JACCOTTET (1998) et (2003), p. 71-100, MASSA (2012).

14. Cf. supra note 4.

15. Sur les distorsions que peut engendrer la prise en compte des fonctions rituelles comme base d'analyse, cf. infra n. 17. 
16. Les astérisques indiquent des fonctions exprimées au travers d'un verbe dans l'inscription :

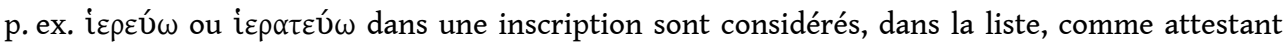

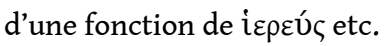

17. Travailler sur les seules fonctions rituelles nous expose certes à certaines distorsions, puisque n'apparaissent dans les documents que les fonctions les plus en vue, concernant des figures importantes de l'association, et le plus souvent de la société, qui se mettent ainsi en avant, au travers des inscriptions. L'existence d'autres fonctions, ou d'autres composantes de telle ou telle association - et notamment la présence des femmes (JAccotTET [2003], I, p. 65-100) - reste possible voire vraisemblable selon les cas de figure; l'existence de traditions dionysiaques thébaines, par exemple, exprimées par un autre biais, oral, rituel, n'est donc pas à exclure. Mais les fonctions rituelles représentent, dans l'état de notre documentation, la trace la plus directe et la plus fiable du fonctionnement véritablement rituel, en marge d'un imaginaire communément diffusé. L'exemple des Bassarai et du thyrsophore d'un groupement éphésien (cf. infra et n. suivante) montrent bien qu'une touche 'traditionnelle' peut être exprimée dans les fonctions, si cela a un sens pour le rituel.

18. IEph 1601-1603 \& 1268 (JACCOTTET $n^{\text {os }} 137-139$ ).

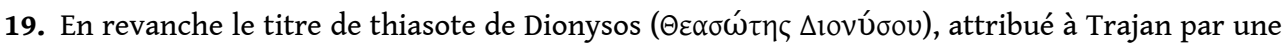
association dionysiaque éphésienne - IEph. 3329 (Jaccottet no 135) - se révèle manifestement honorifique et extérieur à la dynamique proprement rituelle de l'association.

20. Les fragments eschyléens des Bassarai sont issus, en grande partie, des Catastérismes d'Ératosthène, ce qui pose problème pour la reconstruction de l'intrigue de la pièce. Pour une interprétation 'orphique' voir WEST (1983), p. 12-15 qui met l'accent sur le conflit entre Apollon et Dionysos ; contra DI MARCo (1993).

21. Sur les Bassarika de Dionysios voir Agosti (2001).

22. IEph 1211 (JACCOTTET $\mathrm{n}^{\circ}$ 141).

23. IGBulg. 401 (JACCOTTET $\left.n^{\circ} 46\right)$

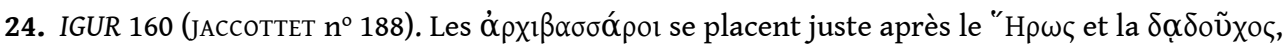
mis en exergue, et les prêtres, dans la hiérarchie de l'association dont la liste redonne le squelette. Les titres des premières fonctions, réservées à un nombre restreint de membres, sont destinés à distinguer les élites familiales du reste de cette double familia bien élargie, cf. JACCOTTET (2003), I, p. 30-53.

25. MORAND (2001), p. 249-282 et 343-346 (inscriptions), JACCOTTET (2003), I, p. 101-122 et II, p. 182-189 (origine et développement).

26. JACCOTTET $\mathrm{n}^{\circ}$ 92-93.

27. Il est clair que nous ne disposons que d'une documentation forcément partielle qui rend, comme toujours, toute vision «statistique » périlleuse. Sans prétendre ainsi reproduire l'entier de la réalité antique, l'examen chronologico-géographique de attestations des boukoloi peut néanmoins mettre en lumière quelques lignes directrices dans leur développement et leur diffusion.

28. La carte répertorie, en les numérotant, tous les lieux où on été trouvées des inscriptions attestant la présence d'une ou de plusieurs associations dionysiaques. Parmi ces occurrences, 11 sites ont livré du matériel évoquant des (archi)boukoloi: no 14 Abdère $\mathrm{III}^{\mathrm{e}} \mathrm{s}$. de notre ère (?)

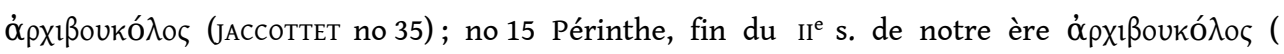

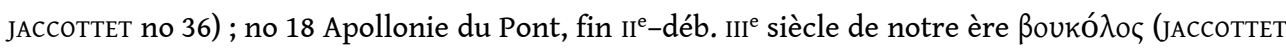

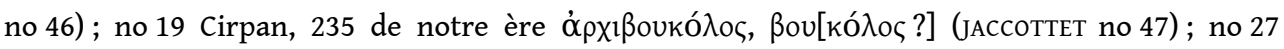
Martviza (Pleven Mésie) archibycolus (JAccotteT no 69); no 38 Ancyra Sidera (Phrygie) III s.

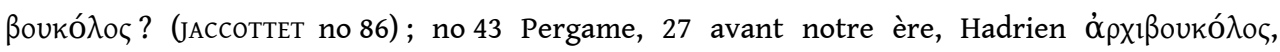

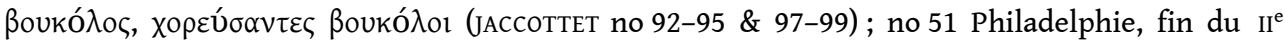

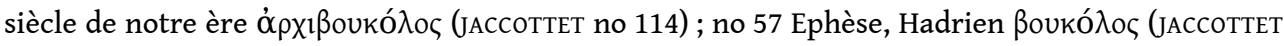


no 138-139) ; no 63 Physcos $2^{\mathrm{e}}$ moitié du II siècle de notre ère ßoukó

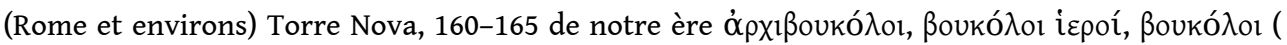
JACCOTTET no 188) ; Rome fin du Iv ${ }^{\mathrm{e}}$ siècle de notre ère archibuolus (JACCOTTET no 192-194) ; no 77 (pas sur la carte) Thamugadis (Timgad) ${ }_{\mathrm{II}}{ }^{\mathrm{e}}$ siècle de notre ère bucolista (JACCOTTET no 198-200).

29. JAссоттет (2003), I, p. 30-53. Thamugadis (Timgad) a également fourni trois inscriptions mentionnant un bucolista à la fin $\mathrm{du}_{\mathrm{II}}^{\mathrm{e}}$ siècle de notre ère, toutes trois funéraires (JACCOTTET $\mathrm{n}^{\circ}$ 198-200). Les relais qui ont amené cette fonction à Thamugadis, ne peuvent être éclaircis en l'état de nos connaissances. Il n'est pas non plus possible de savoir dans quel cadre ces trois hommes ont assumé cette fonction. Seule sa parenté avec les ßoukódor nous incite à en faire des témoignages dionysiaques.

30. JАССОТTЕT (2003), II, p. 319-320.

31. IPergamon 485 (JACCOTTET $\left.n^{\circ} 94\right) \mathrm{I}^{\text {er }}$ siècle de notre ère ( $1^{\text {re }}$ moitié ?).

32. IPergamon 487 (JACCOTTET $n^{\circ}$ 97) fin du I $^{\text {er }}$ siècle de notre ère, et IGR IV 386 (JACCOTTET $n^{\circ} 98$ ) après 106 de notre ère.

33. IGR IV 386 (JACCOTTET $n^{\circ} 98$ ) après 106 de notre ère et IPergamon 486a (JACCOTTET $n^{\circ}$ 99) époque d'Hadrien.

34. Sur la concurrence entre Éphèse et Pergame voir Aelius Aristide, Discours XXIII, 65-67 et HELLER (2006).

35. IEph 3329 (JACCOTTET $\mathrm{n}^{\circ}$ 135).

36. IEph 275 (JACCOTTET $\left.n^{\circ} 136\right)$.

37. IEph 293 (JACCOTTET $\mathrm{n}^{\circ}$ 142).

38. IEph 1601-1603 \& 1268 (JACCOTTET $\mathrm{n}^{\circ} 137-139$ ).

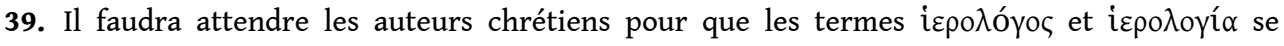
diffusent dans le langage religieux : cf. par exemple le Pseudo-Denys l'Aréopagite dans son traité, La Hiérarchie ecclésiastique, p. 68, 70, 80 et 84 éd. HEIL-RITTER.

40. Cf. GRAF, JOHNSTON (2007), p. 176. Pour une réflexion générale sur la nature des hieroi logoi, voir HENRICHS (2003), p. 231-250 et BAUMGARTEN (1998).

41. Cf. Hérodote II, 48, 3 (cultes dionysiaques) ; 51, 4 (mystères de Samothrace) ; 62, 2 (fête égyptienne) ; 81, 2 (interdiction de l'usage de la laine). Sur l'emploi hérodotéen de l'expression, voir HENRICHS (2003), p. 235-239.

42. Pausanias VIII, 15, 4. Cf. aussi Plutarque, Isis et Osiris, 353d.

43. D'après GRAF, JOHNSTON (2007), p. 180-181 il s'agissait de discours qui circulaient sous une forme écrite garantissant la préservation de leur contenu dans la mesure où ils étaient utilisés par des petits groupes. Contra HENRICHS (2003), p. 240 : « In Greece proper, hieroi logoi remained by definition unwritten. »

44. IEph 1595 (JACCOTTET $\mathrm{n}^{\circ}$ 140).

45. Si l'on choisit, comme nous l'avons fait, de considérer que ce document émane plus probablement des deux entités conjointes que d'une seule.

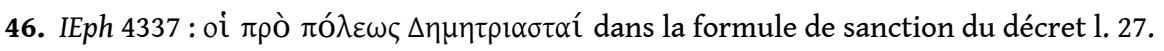

47. On notera par exemple qu'une base de statue éphésienne de la fin du II siècle de notre ère

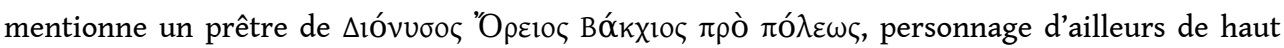
rang social (C. Flavius Furius Aptus, IEph 1267 [Jaccottet $n^{\circ}$ 134]). Ce sont bel et bien les prêtres ou, dans le cas des Démétriastes, les membres d'une communauté qui sont qualifiés de pro poleôs, non directement la divinité.

48. IEph. 1270.

49. Cf. poland (1909) p. 36-41, GRAF (2003), Belayche (2013), p. 35-39, Borgeaud (2013). Pour la diffusion et le sens des mystes dionysiaques, cf. JACCOTTET (2003), I, p. 128-131 et (2006). 
50. «Ces cérémonies appelées $\mu v \sigma \tau \eta ́ p ı \alpha$ apparaissent plutôt comme des moments cérémoniels réservés à des happy fews. [...] Ces fonctions reflètent la hiérarchisation sociale accrue, une 'aristocratisation' au sein même des élites, et la diffusion d'un emploi métaphorique du vocabulaire mystérique, dont la générosité des élites faisait profiter toute la population ", BELAYCHE 2013, p. 39.

51. Pour un répertoire des attestations voir sснUdDEвоом (2009). Sur la large diffusion du vocabulaire mystérique voir GRAF (2003) et BELAYCHE (2013), p. 35-39.

52. Cf. HAMILTON (1977), RIEDWEG (1987), qui suit en partie les positions de NOCK (1952), STROUMSA (1996), p. 20-22 et SFAMENi GASPARRo (2000).

53. Pour le baptême comme $\mu$ $\sigma \tau$ ńpıv cf. Athanase, Discours contre les Ariens XLII, Eusèbe de

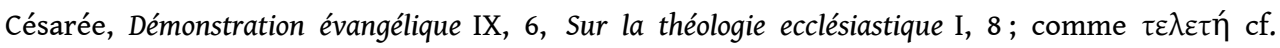
Origène, Contre Celse III, 59 et Jean Chrysostome, À Théodore I, 17. Pour l'eucharistie comme

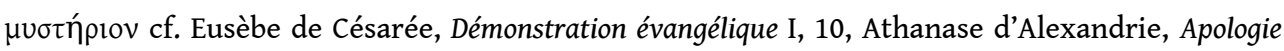
contre les Ariens II, 31, Cyrille d'Alexandrie, Les catéchèses baptismales XII, 23, Épiphane de

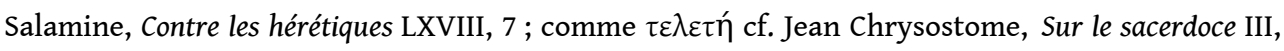
4.

54. Sur le problème de l'authenticité de ses lettres et de la « question ignacienne » voir MUNIER (1993) et PRINZIVAlLI, SimONETTi (2010), p. 283-291. Pour un commentaire détaillé voir schoEdel (1985).

55. Sur la datation des lettres d'Ignace voir FOSTER (2007) et PRINZIVALLI, SIMONETTI (2010), p. 290.

56. Cf. Ignace, Lettre aux Éphésiens VII, 1 : l'auteur parle sans doute de groupes considérés comme « hérétiques».

57. Cf. PRINZIVALLI, SIMONETTI (2010), p. 294.

58. Les textes chrétiens les plus anciens n'utilisent pas tous le terme «chrétien(s)»: dans les écrits qui rentreront dans le Nouveau Testament, il n'est attesté que dans les Actes des Apôtres XI, 26 et XXVI, 21, et dans la Lettre à Pierre IV, 16. En revanche, le terme « chrétien(s) » apparaît dans la Didachè XII, 4 et chez Ignace d'Antioche, Lettre aux Éphésiens XI, 2, Lettre aux Magnésiens IV, 1, Lettre aux Romains III, 2, Lettre à Polycarpe VII, 3.

59. Cf. Ignace, Lettres aux Éphésiens VIII, 2.

60. Cf. HARLAND (2009).

61. Cf. Ignace, Lettre aux Éphésiens IV, 2.

62. Sur la « réforme » d'Ignace on se référera à BRENT (1992).

63. Cf. Ignace, Lettre aux Éphésiens IX, 2.

64. Le corpus des Lettres de Paul (ou qui lui sont attribuées) présente presque exclusivement le

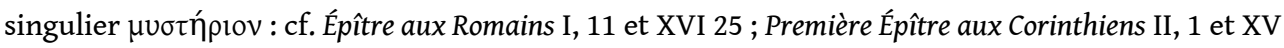
51 ; Épittre aux Éphésiens I, 9, III, 3, 9, V, 32 et VI, 19 ; Épitre aux Colossiens I, 26, 27, II, 2 et IV, 3 ; Épître aux Thessaloniciens II, 7 ; Première Épitre à Timothée III, 9 et 16. Il n'y a que deux occurrences de $\mu v \sigma \tau \eta ́ p l \alpha:$ Première Épittre aux Corinthiens I, 13 et II, 14. Voir NOCK (1952).

65. Cf. Ignace, Lettre aux Éphésiens XII, 2.

66. Cf. par exemple IG XII, 8, 173. Sur le terme $\sigma u \mu \mu u ́ \sigma \tau \alpha l$ voir BURKERT (1987), p. 75.

67. Cf. JAсCотTET no $20,32,35,43,82,89,112,161$.

68. Cf. JACCOTTET (2009) et (2011).

69. Cf. Ignace, Lettre aux Éphésiens IX, 1.

70. Sur le rôle du théâtre et du langage théâtral chez Clément d'Alexandrie voir LUGARESI (2008), p. 489-503.

71. Clément d'Alexandrie, Protreptique aux Hellènes I, $2,3$.

72. JACCOTTET $\mathrm{n}^{\circ}$ 115-118, 120-122 et commentaire p. 216-217.

73. Sur les rapports d'Ignace avec la culture grecque et romaine voir BRENT (2006). Sur les cultes à mystères en particulier voir BRENT (2005). 
74. Le langage utilisé par Ignace varie, ce qui permet de faire l'hypothèse d'un usage spécifique selon la communauté à laquelle l'auteur chrétien s'adressait, cf. TREVETT (1992), p. 75-80 et HARLAND (2009), p. 47.

75. Cette inscription est communément appelée « loi sacrificielle » dans la critique moderne, en

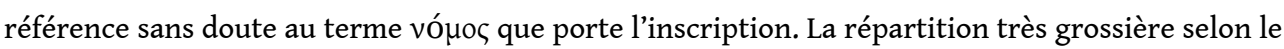
type de mise en place ou de combustion des victimes (190 dont on prélève le cœur et détache la cuisse ; 175 en offrande complète) suffit-elle à faire de ce texte une « loi sacrificielle » ? On y sent plus les termes du contrat implicitement accepté par tout prytane entrant en charge en matière de sacrifices publics et donc de dépenses sacrificielles à concéder pour le bien de la communauté ; les amendes prévues en cas de faute ou de manquement à ces devoirs rituels (1. 2532) soulignent le caractère contractuel du texte; nous renvoyons à PARKER (2004), LUPU (2005), p. 4-9 et CARBON, PIRENNE-DELFORGE (2012) pour la question du terme controversé de «loi sacrée » et à HARRIS (2013) pour les critères juridiques et formels d'identification d'une loi.

76. IEph 10. On notera que PRICE (1999) a choisi de retenir cette inscription dans les 15 documents représentatifs des « religions des anciens Grecs » $\left(n^{\circ} 7\right)$. L'affirmation de la filiation à la tradition est, sans doute, à comprendre comme un acte de communication plus que comme une déclaration à prendre au pied de la lettre. Sur l'importance de la communication et de l'affirmation de la conformité à la tradition lors de modifications rituelles, cf. STAVRIANOPOULOU (2006 b).

77. Ce parallèle nous a été fort judicieusement suggéré par Nicole Belayche lors de l'atelier Chicago-Paris 2012. Qu'elle en soit ici vivement remerciée.

78. Denys d'Halicarnasse, Antiquités romaines II, 73, trad. V. Fromentin, J. SCHNÄBELE, Paris, Les

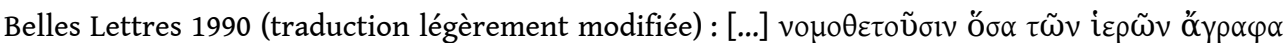

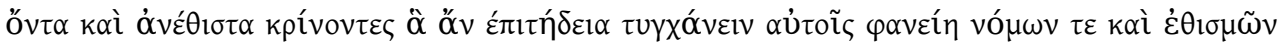

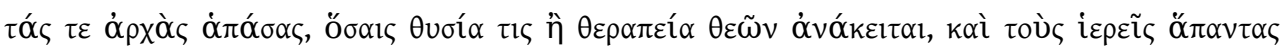

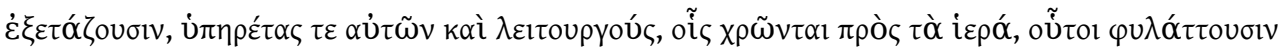

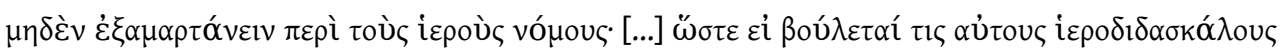

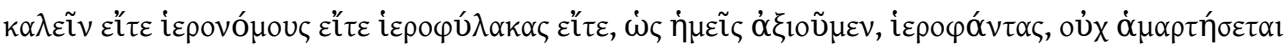

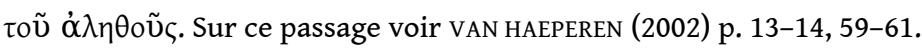

79. On renverra à STAVRIANOPOULOU (2006) et BRICAULt, BONNET (2013 a) pour des pistes de recherche dans cette direction.

80. SCHEID (2010), p. 9 : «Dans la mesure où elle n'est pas écrite, la norme n'est pas connue en détail, et de toute façon ne porte pas sur le détail. Comme le terme ritus, elle désigne une façon de faire, une façon de célébrer le culte, non une série de rites et de règles précises. »

81. Sur le caractère résolument humain des interventions sur les rituels cf, scHEID (2010), part. p. 7 pour le monde romain, STAVRIANOPOULOU (2006 b) pour le monde grec. Sur l'importance de la communication qui donne l'impression d'une immuabilité par delà les changements effectifs, Cf. StAVRianopoulou (2006 c), p. 10 et (2006 b). PiRENNE-DELForge, SCHEID (2013), p. 312 : « De la même manière qu'un système religieux, quel qu'il soit, contient toujours des éléments qui proviennent de divers arrière-plans culturels, ce même système recèle une potentialité dynamique qui ouvre sur des éléments nouveaux, fussent-ils parés des vêtements respectables de la tradition ancestrale. "

82. Dans le seul cadre des associations dionysiaques citons les Thiasites de Callatis, chargés par la ville de la tenue des initiations : pour les inscriptions, cf. AVRAM (1999), $\mathrm{n}^{\circ} 36,42-47$ et 80, JACCOTTET (2003), $\mathrm{n}^{\circ}$ 54-61, pour l'analyse, AVRAM (1995) et (2000), JACCOTTET (2003), I, 150-162, (2005), p. 196-199 et (2006), p. 223-224; à Milet, les prêtres des diverses corporations dionysiaques défilent derrière le prêtre et la prêtresse publics (LSAM $n^{\circ} 38$, JACCOTTET $n^{\circ} 150$ ).

83. Sur la perméabilité du 'privé' et de 'l'officiel' dans le cadre dionysiaque en particulier, cf.

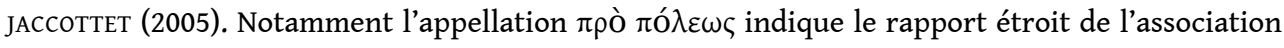


à la cité (à Éphèse, Thasos, Augusta Tajana, Smyrne Magnésie du Méandre, Milo) ; plusieurs associations honorent Dionysos sous l'épiclèse du culte officiel de la cité (Akmonia, Sébasté en Phrygie, Philadelphie en Lydie) ; enfin les honneurs rendus aux empereurs par les associations marquent aussi cette perméabilité basée sur une certaine course à la visibilité (à Éphèse ou Périnthe; sur les relations entre les associations éphésiennes de toute obédience et les empereurs, cf. HARLAND (1996), notamment p. 328 et 331).

\section{RÉSUMÉS}

Trois dossiers distincts, mais complémentaires, et rattachés à la ville d'Éphèse sont convoqués pour éclairer des cas concrets de transmission de pratiques, de savoirs ou de vocabulaire rituels. Tout d'abord, les associations dionysiaques d'Éphèse permettent d'aborder la question des référents à partir desquels se constitue un rituel, vu que chaque association est un cas unique, au profil cultuel particulier. Ensuite, l'examen de la Lettre aux Éphésiens d'Ignace d'Antioche souligne l'utilisation par un des premiers auteurs chrétiens d'un vocabulaire associatif et mystérique pour véhiculer son message chrétien. Cela suppose une large diffusion et une connaissance partagée de pratiques rituelles. Enfin, une inscription d'Éphèse met en lumière le rôle du hiérophante public comme indispensable "passeur de tradition » dans l'exécution des rites publics par les magistrats et insiste sur la séparation des tâches et des savoirs en matière rituelle. La mise en parallèle de ces trois cas permet de remettre en question la distinction entre un savoir proprement cultuel et des connaissances fondées plus largement sur des références culturelles: dans un savoir religieux en action, c'est le rite effectif qui devient lui-même référence culturelle.

Three different - although complementary - dossiers on Ephesus shed light on the question of the transmission of knowledge, practices and vocabulary relating to rituals. First, the analysis of the Dionysiac associations in Ephesus aims at addressing the question of the referents constituting a ritual, since each association is unique, characterized by a specific cultural profile. Secondly, the analysis of the Letter to the Ephesians by Ignatius of Antioch shows how one of the first Christian authors uses the vocabulary used by associations as well as referring to mysteries in order to deliver his Christian message. This presupposes a widespread and shared knowledge of ritual practices. Finally, an inscription from Ephesus underlines the role of the public hierophant as essential passeur de tradition in the magistrates' execution of public rituals and emphasizes the division of tasks and knowledge within the context of these rituals. The parallel study of these three case studies leads us to question the distinction usually made between cultic knowledge and knowledge based on cultural referents: within the context of religious knowledge in action, the actual rite itself becomes a cultural referent.

\section{AUTEURS}

\section{ANNE-FRANÇOISE JACCOTTET}

Université de Genève

anne-francoise.jaccottet@unige.ch 
FRANCESCO MASSA

Aix-Marseille Université

Centre Paul-Albert Février (UMR 7297 CPAF-TDMAM), LabexMed

f.massa@tin.it 